Document downloaded from:

http://hdl.handle.net/10251/150338

This paper must be cited as:

Benavent López, E.; Corberán, A.; Plana, I.; Sanchís Llopis, JM. (2011). New Facets and an Enhanced Branch-and-Cut for the Min-Max K-Windy Rural Postman Problem. Networks. 58(4):255-272. https://doi.org/10.1002/net.20469

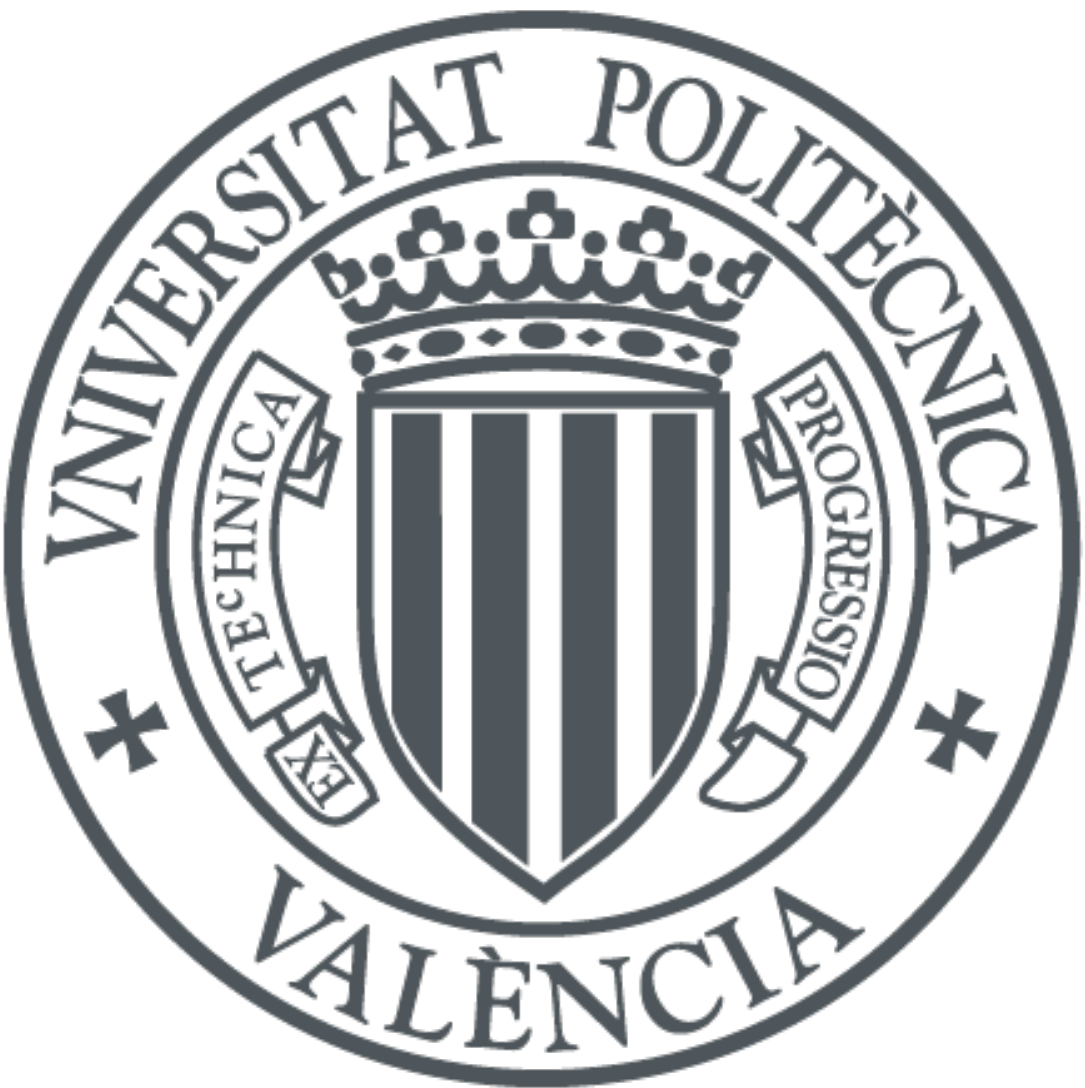

The final publication is available at

https://doi.org/10.1002/net.20469

Copyright WILEY-BLACKWELL

Additional Information 


\title{
New Facets and an enhanced Branch-And-Cut FOR the Min-Max $K$-Vehicles Windy RuRal Postman Problem
}

\author{
Enrique Benavent ${ }^{1}$, Angel Corberán ${ }^{1 *}$, \\ Isaac Plana ${ }^{2}$ and José M. Sanchis ${ }^{3}$ \\ ${ }^{1}$ D.E.I.O., Universitat de València (Spain) \\ ${ }^{2}$ D.M.E.E., Universitat de València (Spain) \\ ${ }^{3}$ D.M.A., Universidad Politécnica de Valencia (Spain)
}

\begin{abstract}
The Min-Max Windy Rural Postman Problem is a multiple vehicle version of the Windy Rural Postman Problem, WRPP, which consists of minimizing the length of the longest tour in order to find a set of balanced tours for the vehicles. In [6], an ILP formulation and a partial polyhedral study was presented, and a preliminary branch-and-cut algorithm that produced some promising computational results was implemented. In this work, we present further results on this problem. We describe several new facet-inducing inequalities obtained from the WRPP, as well as some inequalities that have to be satisfied by any optimal solution. We present an enhanced branch-and-cut algorithm that takes advantage of both these new inequalities and high quality MM $K$-WRPP feasible solutions obtained by a metaheuristic. Computational results on a large set of instances are also reported.
\end{abstract}

Keywords: Rural Postman Problem, Windy Postman Problem, Windy Rural Postman Problem, Facets, multivehicle.

\section{Introduction}

The Rural Postman Problem on a windy graph (WRPP) generalizes most of the single-vehicle Arc Routing Problems. Let $G=(V, E)$ be a 'windy' graph, i.e., an undirected graph with two nonnegative costs $c_{i j}, c_{j i}$ associated with each edge $e=(i, j)$, corresponding to the costs of traversing $e$ from $i$ to $j$ and from $j$ to $i$, respectively. Let $E_{R}$ be a subset of required edges (representing those edges requiring some service to be done along them). The WRPP consists of finding a tour of minimum cost traversing each edge in $E_{R}$ at least once.

In this paper we deal with the situation where there is a fleet of identical vehicles that jointly service the required edges. If the capacity of the vehicles is not restricted and we look for $K$ routes starting and ending at the depot, in such a way that all the edges of the graph are serviced by exactly one vehicle and the total distance is minimized, we have the $K$-CPP

*corresponding author: angel.corberan@uv.es 
which Assad, Pearn and Golden [5] showed to be solvable in polynomial time in the undirected and directed cases. Nevertheless, Pearn [14] proved that the $K$-CPP is $N P$-hard when it is defined on a mixed or windy graph. The Min-Max $K$-CPP is a more realistic problem in which the objective is to minimize the length of the longest route among the $K$ vehicles. This is a way of balancing the working load of the vehicles. It was introduced by Frederickson, Hecht and Kim [12], who also proved that the Min-Max $K$-CPP is $N P$-hard and proposed a $\left(2-\frac{1}{K}\right)$-approximation algorithm. More recently, Ahr and Reinelt present several lower bounds and heuristics for this problem ([2]) and a Tabu Search procedure that produces very good solutions ([3]). Finally, in Ahr [1] some more results on the Min-Max $K$-CPP, including an exact solution method based on a branch-and-cut approach, are presented.

In Benavent et al. [6], the Min-Max $K$-vehicles Windy Rural Postman Problem (MM $K$-WRPP) is introduced. Given a windy graph, a distinguished vertex (the depot), a subset of required edges and a fixed number $K$ of vehicles, the problem consists of finding a set of $\mathrm{K}$ tours for the vehicles in such a way that each tour starts and ends at the depot and each required edge is serviced by exactly one vehicle. The objective is to minimize the length of the longest tour in order to find a set of balanced tours for the vehicles. In that paper, a formulation of the problem is presented and some valid inequalities are introduced. Furthermore, the polyhedron associated with the MM $K$-WRPP solutions is partially described and a branch-and-cut algorithm producing encouraging results on a large set of instances is presented.

In this work, we present further results on this problem. In Section 2, we present some notation, the formulation of the problem and the inequalities studied in [6]. In Section 3 we describe several new facet inducing inequalities obtained from the WRPP and, in Section 4, some inequalities that have to be satisfied by any optimal solution. An enhanced branchand-cut algorithm that uses these new inequalities and high quality MM $K$-WRPP feasible solutions obtained by a metaheuristic is presented in Section 5 . The computational results obtained on a large set of instances are shown in Section 6 and in Section 7 some conclusions are drawn.

\section{The Min-Max $K$-vehicles Windy RPP}

Let $G=(V, E)$ be an undirected and connected graph with two costs $c_{i j}, c_{j i}$ associated with each edge $e=(i, j)$, and assume that node $1 \in V$ represents the depot. Let $E_{R} \subset E$ be the set of required edges. Consider a fixed number of vehicles, which we will denote from now on $\widehat{K}$ in order to avoid confusion with the letter $K$ used for the description of some well known families of inequalities that will be presented in Section 3. We have to find a tour (closed walk starting and ending at the depot) for each vehicle such that each required edge is traversed at least once by at least one vehicle. A set of such tours (one per vehicle) will be called a MM $K$-WRPP solution. The objective is to find a MM $K$-WRPP solution such that the cost of the maximum cost vehicle tour is minimum.

As in [6], we will assume that each vertex in $V$ is incident with at least one required edge. Let us call $G_{R}=\left(V, E_{R}\right)$ the graph induced by the required edges. In general, $G_{R}$ is not connected. The sets of vertices $V_{1}, V_{2}, \ldots, V_{p}$ of the $p$ connected components of $G_{R}$ are called $R$-sets and the subgraphs induced by them are called $R$-connected components. Given two node subsets $S, S^{\prime} \subseteq V,\left(S: S^{\prime}\right)$ denotes the edge set with one end-point in $S$ 
and the other in $S^{\prime}$. Given a node subset, $S \subseteq V$, let us denote $\delta(S)=(S: V \backslash S)$ and let $E(S)=\{(i, j) \in E: i, j \in S\}$ be the set of edges with both endpoints in $S$. The previous sets restricted to the required edges are denoted by $\delta_{R}(S), E_{R}(S)$ and $\left(S: S^{\prime}\right)_{R}$.

To formulate the problem, in [6], $2 \widehat{K}$ variables $x_{i j}^{k}, x_{j i}^{k}$ are defined for each edge $e=(i, j) \in$ $E$, representing the number of times edge $e$ is traversed by vehicle $k$ from $i$ to $j$ or from $j$ to $i$, respectively. In addition, if edge $e$ is required, $\widehat{K}$ more variables $y_{e}^{k}$ which take the value 1 if edge $e$ is serviced by the vehicle $k$ and 0 otherwise, are defined. Finally, an artificial variable $z$ is used to minimize the maximum tour cost. The Min-Max $\widehat{K}$-WRPP can be formulated as follows:

$$
\begin{aligned}
\text { Minimize } & z \\
\text { s.t.: } & \\
\sum_{(i, j) \in E}\left(c_{i j} x_{i j}^{k}+c_{j i} x_{j i}^{k}\right) \leq z & \forall k=1, \ldots, \widehat{K} \\
\sum_{k=1}^{K} y_{e}^{k}=1, & \forall e \in E_{R} \\
x_{i j}^{k}+x_{j i}^{k} \geq y_{e}^{k} & \forall e=(i, j) \in E_{R} \text { and } \forall k=1, \ldots, \widehat{K} \\
\sum_{(i, j) \in \delta(i)}\left(x_{i j}^{k}-x_{j i}^{k}\right)=0, & \forall i \in V \text { and } \forall k=1, \ldots, \widehat{K} \\
x^{k}(\delta(S)) \geq 2 y_{e}^{k}, & \forall S \subset V \backslash\{1\}, \quad \forall e \in E_{R}(S), \quad \forall k=1, \ldots, \widehat{K} \\
x_{i j}^{k}, x_{j i}^{k} \geq 0 \text { and integer } & \forall(i, j) \in E, \quad \forall k=1, \ldots, \widehat{K} \\
y_{e}^{k} \in\{0,1\} & \forall e \in E_{R} \quad \forall k=1, \ldots, \widehat{K}
\end{aligned}
$$

where $x^{k}(F)$ denotes $\sum_{(i, j) \in F}\left(x_{i j}^{k}+x_{j i}^{k}\right)$ for any subset $F \subseteq E$.

Inequalities (1) imply that the maximum cost vehicle route is minimized. Equations (2) assure that each required edge is serviced by exactly one vehicle and traversing inequalities (3) force a vehicle to traverse the edges it services. Symmetry equations (4) force each vehicle route to be symmetric, while connectivity inequalities (5) ensure that each vehicle route connects the edges it services and the depot.

A vector $\left(x^{1}, y^{1}, x^{2}, y^{2}, \ldots, x^{\widehat{K}}, y^{\widehat{K}}\right)$ with $\left(2|E|+\left|E_{R}\right|\right) \widehat{K}$ components satisfying (2) to (7) will be called a solution for the MM $K$-WRPP on $G$. The pair $\left(x^{k}, y^{k}\right)$ is the route associated with vehicle $k$, while $x^{k}$ will denote the corresponding tour. Note that this formulation allows solutions in which a vehicle tour $x^{k}$ is formed by several disconnected subtours, one of them connecting all the edges it services to the depot and the others traversing edges not serviced by this vehicle. Note also that solutions where a given vehicle neither traverses nor services any edges are allowed $\left(x^{k}=y^{k}=0\right)$.

Let $\operatorname{KWRPP}(G)$ be the convex hull of all the MM $K$-WRPP solutions, which is a polyhedron of dimension $\widehat{K}(2|E|-|V|+1)+(\widehat{K}-1)\left|E_{R}\right|$. In [6] it is shown that, if $F(x) \geq c_{0}$ is a valid inequality for the (1 vehicle) WRPP defined on graph $G$, then inequality $\sum_{k} F\left(x^{k}\right) \geq c_{0}$, called aggregate inequality, is valid for $\operatorname{KWRPP}(G)$. Hence, aggregate R-odd cut, K-C, Hon- 
eycomb and other inequalities for the WRPP described in Corberán, Plana and Sanchis [10] are valid inequalities for $\operatorname{KWRPP}(G)$.

In $[6]$ it is also shown that the following inequalities are facet-inducing for $\operatorname{KWRPP}(G)$ :

- trivial inequalities, $x_{i j}^{k} \geq 0, x_{j i}^{k} \geq 0 \forall(i, j) \in E$ and $\forall k$ and $y_{e}^{k} \geq 0 \forall e \in E_{R}$ and $\forall k$ (inequalities $y_{e}^{k} \leq 1$ for $e \in E_{R}$ are implied by $y_{e}^{k} \geq 0$ and the system equations (2)),

- traversing inequalities (3), $x_{i j}^{k}+x_{j i}^{k} \geq y_{e}^{k}, \forall e=(i, j) \in E_{R}$ and $\forall k$,

- connectivity inequalities (5), $x^{k}(\delta(S)) \geq 2 y_{e}^{k}, \forall S \subset V \backslash\{1\}$ and $e \in E_{R}(S)$ and $\forall k$,

- aggregate $R$-odd cut inequalities, $\sum_{k=1}^{\widehat{K}} x^{k}(\delta(S)) \geq\left|\delta_{R}(S)\right|+1, \forall S \subset V$ such that $\left|\delta_{R}(S)\right|$ is odd,

- parity inequalities, $x^{k}(\delta(S)) \geq 2 y^{k}(F)-|F|+1, \forall S \subset V, \forall F \subset \delta_{R}(S)$ such that $|F|$ is odd, and $\forall k$, and

- P-aggregate parity inequalities, $x^{k_{1}}(\delta(S))+\cdots+x^{k_{P}}(\delta(S)) \geq 2 y^{k_{1}}(F)+\cdots+2 y^{k_{P}}(F)-$ $|F|+1, \forall S \subset V, \forall F \subset \delta_{R}(S)$ such that $|F|$ is odd, and for every subset $\left\{k_{1}, k_{2}, \ldots, k_{P}\right\}$ of $P<\widehat{K}$ vehicles.

\section{More facet inducing inequalities}

In this section, we will present new families of facet-inducing inequalities for the polyhedron associated with the MM $K$-WRPP.

$\mathrm{K}-\mathrm{C}$ and $\mathrm{K}-\mathrm{C}_{02}$ inequalities are well known families of facet-inducing inequalities for the WRPP ([10]). In this section we show that the new versions of these families corresponding to a single vehicle, a subset of vehicles and all the vehicles, called disaggregate, P-aggregate and aggregate versions respectively, are valid and facet-inducing inequalities for $\operatorname{KWRPP}(G)$. Similar results regarding its validity have been obtained for the well known Honeycomb inequalities.

\section{1 $\mathrm{K}-\mathrm{C}$ and $\mathrm{K}-\mathrm{C}_{02}$ inequalities}

Although $\mathrm{K}-\mathrm{C}$ and $\mathrm{K}-\mathrm{C}_{02}$ inequalities for the WRPP differ in the coefficients of the variables, they share the same underlying configuration (see Figure 1). It is defined by a partition of $V$, $\left\{M_{0}, M_{1}, M_{2}, \ldots, M_{K-1}, M_{K}\right\}$, such that each $R$-set $V_{i}$ is contained in one of the node sets $M_{0} \cup M_{K}, M_{1}, M_{2}, \ldots, M_{K-1}$, the induced subgraphs $G\left(M_{i}\right)$ are connected and $\left(M_{0}: M_{K}\right)$ contains a positive and even number of required edges. Furthermore, it is assumed that sets $\left(M_{i}: M_{i+1}\right)$ are non-empty. The coefficients $a_{i j}$ of the variables in the inequality are shown in Figure 1, where each number represents the coefficient of the variable associated with the traversal of the edge from the nearest node to the farthest one. The coefficient of a variable associated with an edge not shown in Figure 1 between sets $M_{i}$ and $M_{j}$, can be calculated as the cost of the shortest path from $M_{i}$ to $M_{j}$ using the coefficients shown in this figure. Then, the corresponding $\mathrm{K}-\mathrm{C}$ and $\mathrm{K}-\mathrm{C}_{02}$ inequalities are:

$$
F(x)=\sum_{(i, j) \in E}\left(a_{i j} x_{i j}+a_{j i} x_{j i}\right) \geq 2(K-1)+\alpha_{R}
$$


where $\alpha_{R}=(K-2)\left|\left(M_{0}: M_{K}\right)_{R}\right|$ for the K-C inequality and $\alpha_{R}=(K-1)\left|\left(M_{0}: M_{K}\right)_{R}\right|$ for the $\mathrm{K}-\mathrm{C}_{02}$. Since $\mathrm{K}-\mathrm{C}$ and $\mathrm{K}-\mathrm{C}_{02}$ inequalities are facet-inducing for $\operatorname{WGRP}(G)$ when $\widehat{K} \geq 3$ and $\widehat{K} \geq 2$, respectively, we will assume in this paper that these conditions for $\widehat{K}$ are satisfied.
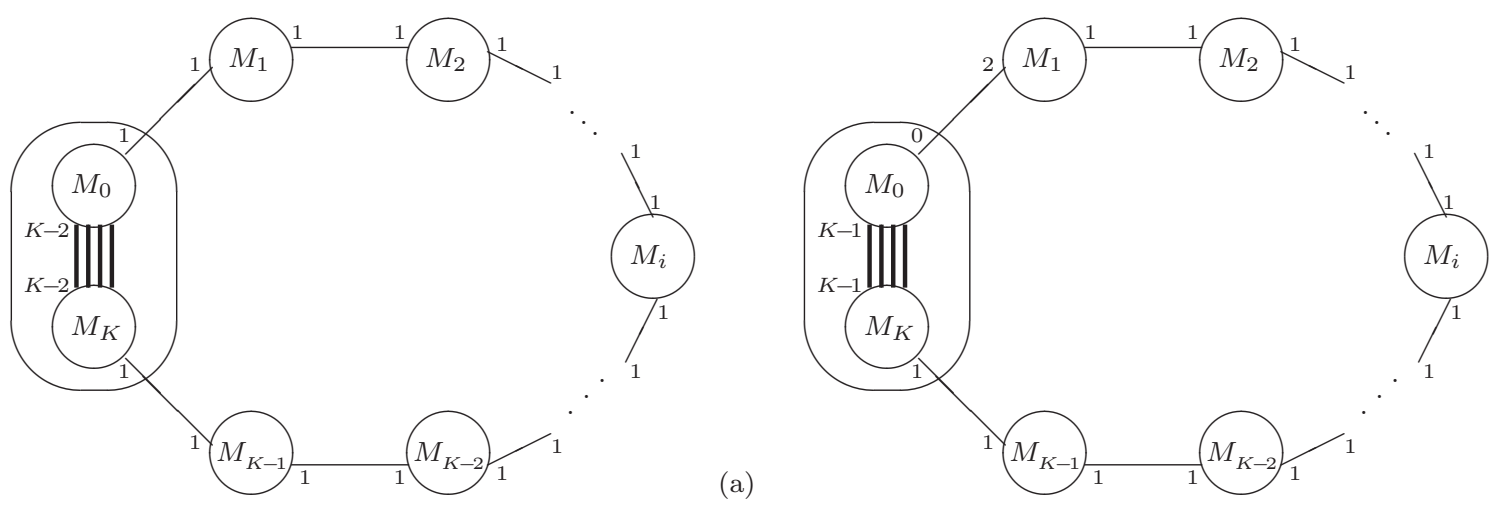

(b)

Figure 1: K-C and $\mathrm{K}-\mathrm{C}_{02}$ configurations.

Then, the corresponding aggregate $\mathrm{K}-\mathrm{C}$ and $\mathrm{K}-\mathrm{C}_{02}$ inequalities are

$$
\sum_{k=1}^{\widehat{K}} F\left(x^{k}\right) \geq 2(K-1)+\alpha_{R},
$$

and, as it has been mentioned before, are valid for the MM $K$-WRPP. In what follows we will prove that they are also facet-inducing.

Note 1 There are two types of WRPP tours that satisfy a K-C inequality with equality, and they will be used in the proof of some theorems:

(i) tours that use the path $M_{0}, M_{1}, \ldots, M_{K-1}, M_{K}$ once (either from $M_{0}$ to $M_{K}$ or viceversa), and traverse $\left|\left(M_{0}: M_{K}\right)_{R}\right|+1$ times the edges in $\left(M_{0}: M_{K}\right)$, and

(ii) tours that use the path $M_{0}, M_{1}, \ldots, M_{K-1}, M_{K}$ twice, once in each direction, except for one pair $\left(M_{i}, M_{i+1}\right), i \in\{0,1, \ldots, K-1\}$, and traverse $\left|\left(M_{0}: M_{K}\right)_{R}\right|$ times the edges in $\left(M_{0}: M_{K}\right)$

Theorem 1 Aggregate $K-C$ inequalities (9) are facet-inducing for $K W R P P(G)$ if the following conditions are satisfied:

1. The depot $1 \in M_{0} \cup M_{K}$ and

2. $\left|E_{R}\left(M_{i}\right)\right|=1$ for each $i=1,2, \ldots, K-1$.

Proof: To simplify this proof and later ones, we will consider the case $\widehat{K}=3$, although they can be easily extended to any value of $\widehat{K}$.

Consider the (1 vehicle) WRPP defined on $G$ and let $\operatorname{WRPP}(G)$ denote its associated polyhedron. It is known ([10]) that, if $G$ is connected, $\operatorname{dim}(\operatorname{WRPP}(G))=2|E|-|V|+1$. Let us call $m=\operatorname{dim}(\operatorname{WRPP}(G))$. Since $\operatorname{dim}(\operatorname{KWRPP}(G))=\widehat{K} m+(\widehat{K}-1)\left|E_{R}\right|([6])$, this is the 
number of MM $K$-WRPP solutions satisfying the inequality with equality we have to find. Given that the K-C inequality $F(x) \geq \alpha=2(K-1)+\alpha_{R}$ is facet-inducing for $\operatorname{WRPP}(G)$, there are $m$ linearly independent tours $w_{1}, w_{2}, \ldots, w_{m}$ for the WRPP on $G$ satisfying $F(x)=\alpha$. Hence, we can build MM $K$-WRPP solutions in the following way. One vehicle performs any WRPP tour $w_{j}$ above and services all the required edges while the other vehicles do nothing. We can define these MM $K$-WRPP solutions arranged as rows of the blocks (a), (b) and (c) in the matrix shown in Figure 2, where a block with a large 0 (or 1 or -1) represents a submatrix with all its entries 0 (or 1 or -1 ) and $I$ represents the identity matrix.

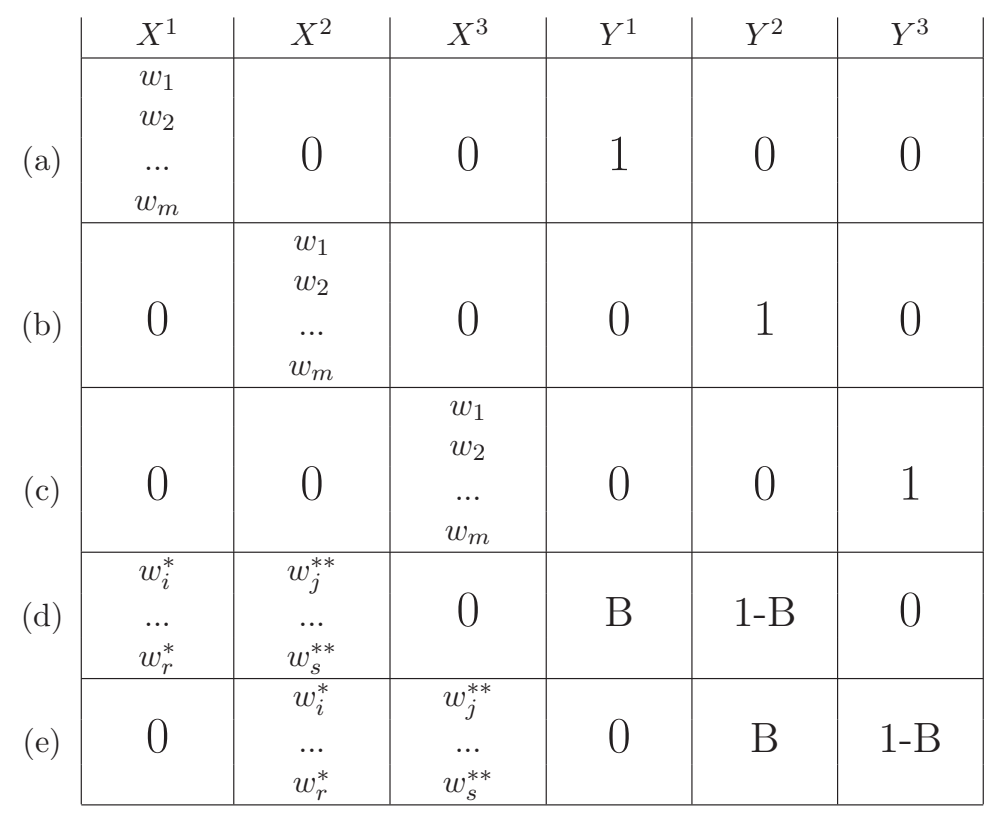

Figure 2: MM $K$-WRPP solutions satisfying $\sum_{k=1}^{\widehat{K}} F\left(x^{k}\right)=2(K-1)+\alpha_{R}$.

To prove that the aggregate K-C inequality is facet-inducing, we need to find $(\widehat{K}-1)\left|E_{R}\right|$ more solutions satisfying (9) with equality. Then, for each pair of vehicles $k, k+1$, we will construct a MM $K$-WRPP solution associated with each required edge $e \in E_{R}$, where vehicle $k$ will service only edge $e$ (when possible), and vehicle $k+1$ will service all the other required edges. Let us suppose that $1 \in M_{0}$.

- For each edge $e \in E_{R}\left(M_{0}\right)$, tour $w^{*}$ leaves the depot, traverses edge $e$ and comes back to the depot, using only edges in $G\left(M_{0}\right)$, while $w^{* *}$ is any tour of types (i) or (ii) described in Note 1 . Vehicle $k$ services only edge $e$ and vehicle $k+1$ services all the remaining required edges.

- For each edge $e \in\left(M_{0}: M_{K}\right)_{R}$, tour $w^{*}$ uses only edges in $G\left(M_{0}\right)$ and $G\left(M_{k}\right)$, and traverses edge $e$ twice, once in each direction, while $w^{* *}$ is any tour such that $w^{*}+w^{* *}$ is a WRPP tour of type (i). Vehicle $k$ services only edge $e$ and vehicle $k+1$ services all the remaining required edges.

- Let $\bar{e}$ be a required edge in $\left(M_{0}: M_{K}\right)_{R}$. For each edge $e \in E_{R}\left(M_{K}\right)$, tour $w^{*}$ traverses edge $e$, edge $\bar{e}$ twice and edges in $G\left(M_{0}\right)$ and $G\left(M_{k}\right)$, while $w^{* *}$ is any tour such that $w^{*}+w^{* *}$ is a WRPP tour of type (i). Vehicle $k$ services edges $e$ and $\bar{e}$ and vehicle $k+1$ services all the remaining required edges. 
- Since $\left|E_{R}\left(M_{i}\right)\right|=1$, we will denote by $\left\{e_{i}\right\}=E_{R}\left(M_{i}\right)$. Then, for each edge $e_{s} \in$ $E_{R}\left(M_{s}\right), s=1,2, \ldots, K-1$, we construct a tour $w^{*}$ traversing edges $e_{1}, e_{2}, \ldots, e_{s}$ in both directions, using the path $M_{0}, M_{1}, \ldots, M_{s}$ once in each direction and edges in $G\left(M_{0}\right)$, while $w^{* *}$ is any tour such that $w^{*}+w^{* *}$ is a WRPP tour of type (ii). Vehicle $k$ services edges $e_{1}, e_{2}, \ldots, e_{s}$, while vehicle $k+1$ services all the remaining required edges.

For the case $\widehat{K}=3$, we obtain rows (d) and (e) of matrix shown in Figure 2, where B is the matrix $\bar{B}$ depicted in Figure 3 after removing the last column and the last row. In order to see that the matrix shown in Figure 2 has full rank we proceed as follows. Given that the $m$ vectors $w_{1}, w_{2}, \ldots, w_{m}$ satisfy $F(x)=\alpha$, if we compute $F\left(x^{1}\right)$ on the columns corresponding to the variables $X^{1}$ and divide this sum by $\alpha$ we obtain a column with all its entries in (a) equal to 1. By subtracting this column from the columns corresponding to $Y^{1}$ we obtain a 0 block in the rows in (a) and columns corresponding to $Y^{1}$. We proceed in the same way to obtain 0 blocks in the rows (b) and (c) and columns corresponding to $Y^{2}$ and $Y^{3}$. Obviously, blocks B and 1-B in (d) and (e) also change. Given the structure of the matrix in Figure 2, if we prove that the matrix in which $\mathrm{B}$ is transformed is non singular, the proof will be complete for any number $\widehat{K}$ of vehicles.

\begin{tabular}{|c|c|c|c|c|c|c|}
\hline$E_{R}\left(M_{0}\right)$ & $\bar{e}\left(M_{0}: M_{K}\right)_{R}$ & $E_{R}\left(M_{K}\right)$ & $e_{1}, e_{2}$ & & $K-1$ & $\mathrm{~F}\left(w^{*}\right)$ \\
\hline I & 0 & 0 & \multicolumn{3}{|c|}{0} & 0 \\
\hline 0 & I & 0 & \multicolumn{3}{|c|}{0} & $\begin{array}{c}\frac{2(K-2)}{\alpha} \\
\vdots \\
\vdots \\
2(K-2)\end{array}$ \\
\hline 0 & $\begin{array}{lll}1 & \\
1 & \\
\vdots & 0 \\
1 & \end{array}$ & I & \multicolumn{3}{|c|}{0} & $\begin{array}{c}\frac{2(K-2)}{\alpha} \\
\vdots \\
\vdots \\
2(K-2) \\
\end{array}$ \\
\hline 0 & 0 & 0 & $\begin{array}{ll}1 & \\
1 & 1 \\
& \\
1 & 1 \\
1 & 1\end{array}$ & $\begin{array}{l}\ddots \\
1 \\
1\end{array}$ & $\begin{array}{ll}1 & \\
1 & 1\end{array}$ & $\begin{array}{l}2 / \alpha \\
4 / \alpha\end{array}$ \\
\hline-1 & -1 & -1 & & -1 & & -1 \\
\hline
\end{tabular}

Figure 3: Matrix $\bar{B}$ of Theorem 1 .

From each column of B in (d) we have subtracted a column obtained as the result of computing $F\left(x^{1}\right)$ on the columns of (d) corresponding to the variables $X^{1}$ divided by $\alpha$. From the construction of tours $w^{*}$ corresponding to (d), the resulting column has a value 0 in the entries associated with the edges in $M_{0}$, a value $\frac{2(K-2)}{\alpha}$ in those associated with $\left(M_{0}: M_{K}\right) \cup M_{K}$ and values $\frac{2}{\alpha}, \frac{4}{\alpha}, \frac{6}{\alpha}, \ldots, \frac{2(K-1)}{\alpha}$ in those associated with edges $e_{1}, e_{2}, \ldots e_{K-1}$, respectively. $\mathrm{F}\left(w^{*}\right)$ will represent this column. Let us suppose $\mathrm{B}$ is a $n \times n$ matrix and let $B^{1}, B^{2}, \ldots, B^{n}$ denote its $n$ columns. We have to prove that the $n$ columns $B^{1}-\mathrm{F}\left(w^{*}\right)$, 
$B^{2}-\mathrm{F}\left(w^{*}\right), \ldots, B^{n}-\mathrm{F}\left(w^{*}\right)$ are linearly independent, i.e., the $n+1$ columns $B^{1}, B^{2}, \ldots, B^{n}$, $\mathrm{F}\left(w^{*}\right)$ are affinely independent. To see this, we append an entry -1 to each one of these vectors and we obtain the $(n+1) \times(n+1)$ matrix $\bar{B}$ depicted in Figure 3. After performing Gaussian elimination on the entries of the last column of matrix $\bar{B}$ it can be seen that it has full rank if, and only if, $K \neq 2$.

In what follows we will see that aggregate $\mathrm{K}-\mathrm{C}$ inequalities (9) are not facet-inducing for $\operatorname{KWRPP}(G)$ if conditions 1 or 2 are not satisfied. We present a new class of aggregate K-C inequalities that dominates the former ones in this case .

Let us consider a K-C configuration in which the depot is in any set in $\left\{M_{0} \cup\right.$ $\left.M_{K}, M_{1}, \ldots, M_{K-1}\right\}$ and there is another one of these sets, different from the one containing the depot, that contains more than one required edge. Let $e$ and $f$ be two such required edges. We will call lifted aggregate K-C inequalities to:

$$
\sum_{k=1}^{\widehat{K}} F\left(x^{k}\right) \geq 2(K-1)+\alpha_{R}+\sum_{k \in I_{1}} 2 y_{e}^{k}+\sum_{k \in I_{2}} 2 y_{f}^{k}-2
$$

where $I_{1}, I_{2}$ is a proper partition of the vehicles index set $\{1,2, \ldots, \widehat{K}\}$.

Theorem 2 Lifted aggregate $K-C$ inequalities (10) are valid for $K W R P P(G)$

Proof: Note that the maximum value for the RHS of (10) is $2(K-1)+\alpha_{R}+2$, which is only achieved when a vehicle in set $I_{1}$ services edge $e$ and a vehicle in set $I_{2}$ services edge $f$. Let us suppose that the depot 1 is in $M_{0} \cup M_{K}$ and $e, f \in E_{R}\left(M_{i}\right)$. In this case two different vehicles travel to set $M_{i}$ and, as it can be seen in Figure $4, \sum_{k=1}^{\widehat{K}} F\left(x^{k}\right) \geq 2(K-1)+\alpha_{R}+2$ holds. In all the other cases, the RHS of (10) is $2(K-1)+\alpha_{R}$ or fewer and then (10) is satisfied.
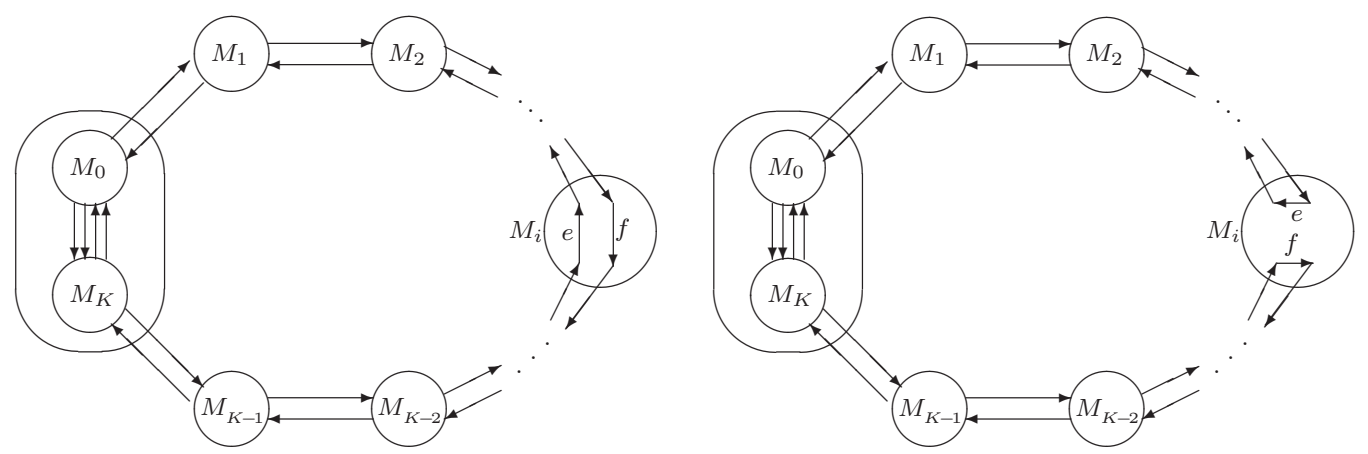

Figure 4: Tours with two vehicles visiting $M_{i}$.

These new inequalities dominate the standard aggregate $\mathrm{K}-\mathrm{C}$ inequalities (9) because if we add the two following valid inequalities

$$
\sum_{k=1}^{M} F\left(x^{k}\right) \geq 2(K-1)+\alpha_{R}+\sum_{i \in I_{1}} 2 y_{e}^{i}+\sum_{j \in I_{2}} 2 y_{f}^{j}-2
$$




$$
\sum_{k=1}^{M} F\left(x^{k}\right) \geq 2(K-1)+\alpha_{R}+\sum_{i \in I_{1}} 2 y_{f}^{i}+\sum_{j \in I_{2}} 2 y_{e}^{j}-2
$$

we obtain inequality (9). Hence, the standard aggregate $\mathrm{K}-\mathrm{C}$ inequalities (9) are not facetinducing for $\operatorname{KWRPP}(G)$ if the depot is in $M_{0} \cup M_{K}$ and $\left|E_{R}\left(M_{i}\right)\right|>1$ holds for any $i=1,2, \ldots, K-1$.

On the other hand, if condition $1 \in M_{0} \cup M_{K}$ is not satisfied, that is, if $1 \in M_{1}$, for example, then we select two required edges $e$ and $f$ from one of the sets $E_{R}\left(M_{0} \cup M_{K}\right)$, $E_{R}\left(M_{2}\right), E_{R}\left(M_{3}\right), \ldots E_{R}\left(M_{K-1}\right)$, and inequality (10) holds. Notice that from $E_{R}\left(M_{0} \cup M_{K}\right)$ we can always select two required edges. Hence, the standard aggregate K-C inequalities (9) are not facet inducing if the depot is not located in $M_{0} \cup M_{K}$.

Theorem 3 Lifted aggregate $K$-C inequalities (10) are facet-inducing for $K W R P P(G)$

Proof: For $M=3$, any partition $I_{1}, I_{2}$ of set $\{1,2,3\}$ contains one and two vehicle indices, respectively. Let us suppose that $I_{1}=\{1,3\}$ and $I_{2}=\{2\}$. Let us suppose also that the depot is in one of the sets $M_{0} \cup M_{K}, M_{1}, M_{2}, \ldots, M_{K-1}$, say $M_{i}$, and that edges $e, f$ are in another different set, say $M_{j}$. The proof is similar to that of Theorem 1. Given that K-C inequality $F(x) \geq \alpha=2(K-1)+\alpha_{R}$ is facet inducing for $\operatorname{WRPP}(G)$, there exist $m$ linearly independent tours $w_{1}, w_{2}, \ldots, w_{m}$ for the WRPP on $G$ satisfying $F(x)=\alpha$. Hence, the vectors represented in the rows of the first 3 blocks of matrix shown in Figure 2 are feasible MM $K$-WRPP solutions satisfying the inequality as an equality (note that all these solutions satisfy $\sum_{k=1}^{M} F\left(x^{k}\right)=\alpha$ because all the vectors $x^{k}$ are zero except one of them, say $x^{p}$, which satisfies $F\left(x^{p}\right)=\alpha$, and the RHS is equal to $\alpha+2-2$ because exactly one variable $y_{e}^{p}$ or $y_{f}^{p}$ is one and all the other are zero).

The vectors represented in the last two block rows of matrix shown in Figure 2 are feasible MM $K$-WRPP solutions satisfying the inequality as an equality using two vehicles, one in $I_{1}$ and the other in $I_{2}$ (vehicles 1 and 2 in (d) and vehicles 2 and 3 in (e)), performing tours $w^{*}$ and $w^{* *}$ and servicing the required edges according to matrix B shown in the top left block in Figure 5. In the first solution, vehicle 1 only services edge $e$. In the following solutions except the last one, vehicle 1 services edge $e$ and exactly one of the remaining required edges except edge $f$. In all these solutions, vehicle 1 performs a tour $w^{*}$ consisting of traveling around the $\mathrm{K}-\mathrm{C}$ structure and, hence, $F\left(w^{*}\right)=2(K-1)$ holds. In the last solution, vehicle 1 services both edges $e, f$ and all the remaining required edges except those in a set next to set $M_{i}$, say $M_{i+1}$, different from $M_{j}$. In this case, tour $w^{*}$ is of type (ii) described in Note 1 except that it does not travel between $M_{i}$ and $M_{i+1}$ (this is done by vehicle 2). Hence, $F\left(w^{*}\right)=\alpha-2$ in this case. We obtain the matrix $\bar{B}$ in Figure 5 and we proceed as in Theorem 1 to show that the matrix obtained from $\mathrm{B}$ after transforming into zeros the 1 block is full rank.

For other values on the number of vehicles $\widehat{K}$ it can be seen that we always can order the vehicles in such a way the part of the matrix formed by the $(M-1) \times(M-1)$ blocks corresponding to matrices B and 1-B (rows (d) and (e) and columns $Y^{1}$ and $Y^{2}$ of the matrix in Figure 2) is upper triangular by blocks with all the diagonal blocks equal to B. For example, if $M=7, I_{1}$ has 5 vehicles associated with edge $e$ and $I_{2}$ has 2 vehicles associated with edge $f$, we can suppose the vehicles are sorted in such a way that vehicles $1,2,3,4,5,6$ and 7 are associated with edges $e, f, e, e, e, e$ and $f$, respectively. Then, we select the $M-2$ pairs of vehicles $(1,2),(2,3),(3,7),(4,7),(5,7)$ and $(6,7)$ to define the solutions described 
with $w^{*}$ and $w^{* *}$.

\begin{tabular}{|cccccc|c|}
$e$ & & $E_{R}$ & & $f$ & $\mathrm{~F}\left(w^{*}\right)$ \\
\hline 1 & & & & & & $2(K-1) / \alpha$ \\
1 & 1 & & & & & $2(K-1) / \alpha$ \\
1 & 0 & 1 & & 0 & & $2(K-1) / \alpha$ \\
& & & $\ddots$ & & & $\vdots$ \\
1 & 0 & 0 & $\ldots$ & 1 & & $2(K-1) / \alpha$ \\
1 & 0 & 1 & $\ldots$ & 1 & 1 & $(\alpha-2) / \alpha$ \\
\hline-1 & -1 & -1 & $\ldots$ & -1 & -1 & -1 \\
\hline
\end{tabular}

Figure 5: Matrix $\bar{B}$ of Theorem 3 .

In summary, given a K-C configuration on graph $G$, if we can select two required edges $e, f$ with its vertices in one of the sets $M_{0} \cup M_{K}, M_{1}, \ldots, M_{K-1}$ except the set containing the depot then the corresponding lifted aggregate K-C inequality (10) is facet-inducing for $\operatorname{KWRPP}(G)$. If it is not possible to select such edges $e, f$ then conditions 1 and 2 of theorem 1 hold and the standard aggregate K-C inequality (9) is facet-inducing for $\operatorname{KWRPP}(G)$.

For aggregate $\mathrm{K}-\mathrm{C}_{02}$ inequalities the situation is different. In addition to the two types (i) and (ii) of WRPP tours described in Note 1 for the standard K-C inequalities (in the case (i) using the path $M_{0}, M_{1}, \ldots, M_{K-1}, M_{K}$ only from $M_{0}$ to $M_{K}$ ), we have another type of WRPP tour:

Note 2 The following WRPP tours satisfy a $\mathrm{K}-\mathrm{C}_{02}$ inequality as an equality:

(iii) tours that use the path $M_{0}, M_{1}, \ldots, M_{K-1}, M_{K}$ twice from $M_{0}$ to $M_{K}$ and traverse half plus one of the edges in $\left(M_{0}: M_{K}\right)_{R}$ from $M_{K}$ to $M_{0}$ and half minus one from $M_{0}$ to $M_{K}$

Then, there exist MM $K$-WRPP solutions satisfying the aggregate $\mathrm{K}_{-} \mathrm{C}_{02}$ inequality $(9)$ as an equality in which two different vehicles traverse the path $M_{0}, M_{1}, \ldots, M_{K-1}, M_{K}$. Hence, there is no need for 'lifting' inequalities.

Theorem 4 Aggregate $K-C_{02}$ inequalities (9) are facet-inducing for $K W R P P(G)$

Proof: The proof is similar to that of Theorems 1 and 3, specially in respect to the MM $K$ WRPP solutions represented in the first 3 blocks of matrix shown in Figure 2. The vectors represented in the rows of the last two blocks of this matrix are feasible MM $K$-WRPP solutions satisfying the inequality as an equality using two vehicles performing tours $w^{*}$ and $w^{* *}$, respectively.

Tour $w^{*}$ consists of traveling around the K-C structure, using the path $M_{0}, M_{1}, \ldots, M_{K-1}, M_{K}$ once and traversing exactly one edge in $\left(M_{0}: M_{K}\right)_{R}$, from $M_{K}$ to $M_{0}$, hence satisfying $F\left(w^{*}\right)=2(K-1)$. Tour $w^{* *}$ uses the path $M_{0}, M_{1}, \ldots, M_{K-1}, M_{K}$ once and traverses all the remaining edges in $\left(M_{0}: M_{K}\right)_{R}$, in such a way that $w^{*}+w^{* *}$ is a WRPP tour of type (iii) described in Note 2. For each required edge $e \in E_{R}$ we have a MM $K$-WRPP solution in which vehicle 1 only services edge $e$ and vehicle 2 
services all the remaining required edges. We obtain the matrix $\bar{B}$ in Figure 6 and proceeding as in Theorem 1, we show that the matrix obtained from B after transforming into zeros the 1 blocks is full rank unless $\left|\left(M_{0}: M_{K}\right)_{R}\right|=2=\left|E_{R}\right|$, which is a nonsense.

\begin{tabular}{|cccccc|c|} 
& & & $E_{R}$ & & & $\mathrm{~F}\left(w^{*}\right)$ \\
\hline 1 & & & & & & $2(K-1) / \alpha$ \\
& 1 & & & & & $2(K-1) / \alpha$ \\
& & 1 & & 0 & & $2(K-1) / \alpha$ \\
& & & $\ddots$ & & & $\vdots$ \\
& 0 & & & 1 & & $2(K-1) / \alpha$ \\
& & & & & 1 & $2(K-1) / \alpha$ \\
\hline-1 & -1 & -1 & $\ldots$ & -1 & -1 & -1 \\
\hline
\end{tabular}

Figure 6: Matrix $\bar{B}$ of Theorem 4 .

Now we deal with the disaggregate K-C inequality corresponding to a single vehicle $k$. Note that the RHS of a K-C inequality for the WRPP, $2(K-1)+(K-2)\left|\left(M_{0}: M_{K}\right)_{R}\right|$, derives from the fact that all the WRPP tours are 'required' to traverse the edges in $\left(M_{0}: M_{K}\right)_{R}$ and to visit the sets $M_{1}, M_{2}, \ldots, M_{K-1}$. Let $\left\{M_{0}, M_{1}, M_{2}, \ldots, M_{K-1}, M_{K}\right\}$ be a partition of $V$ (where each subgraph $\mathrm{G}\left(M_{i}\right)$ is connected) and and let us assume there exists an edge set $F \subseteq\left(M_{0}: M_{K}\right)_{R}$ such that $|F|$ is positive and even and another set $H=\left\{e_{1}, e_{2}, \ldots, e_{K-1}\right\} \subset$ $E_{R}$ such that each $e_{i} \in E\left(M_{i}\right)$. For each vehicle $k$ we will call disaggregate K-C inequality to:

$$
F\left(x^{k}\right) \geq 2 y^{k}(H)+(K-2)\left(2 y^{k}(F)-|F|\right)
$$

and disaggregate $\mathrm{K}-\mathrm{C}_{02}$ inequality to:

$$
F^{\prime}\left(x^{k}\right) \geq 2 y^{k}(H)+(K-1)\left(2 y^{k}(F)-|F|\right)
$$

where $F(x)$ and $F^{\prime}(x)$ are, respectively, the LHS of the standard $\mathrm{K}-\mathrm{C}$ and $\mathrm{K}-\mathrm{C}_{02}$ inequalities corresponding to that configuration.

Theorem 5 Disaggregate $K$-C inequalities (11) and disaggregate $K-C_{02}$ inequalities (12) are valid for $K W R P P(G)$

Proof: We will prove the theorem for inequalities (11). The proof for inequalities (12) is similar. If vehicle $k$ services all the edges in $F \cup H$ then $y^{k}(H)=K-1, y^{k}(F)=|F|$ and the RHS of inequality (11) is $2(K-1)+(K-2)|F|$. In this case, vehicle $k$ must traverse the edges in $F$ (with cost, at least, $(K-2)|F|$ ) and visit the sets $M_{1}, M_{2}, \ldots, M_{K-1}$ (with cost, at least, $2(K-1))$ and $F\left(x^{k}\right) \geq 2(K-1)+(K-2)|F|$ holds.

If vehicle $k$ services all the edges in $H$ except one of them and all the edges in $F$ then vehicle $k$ must traverse the edges in $F$ and visit the sets $\left\{M_{1}, M_{2}, \ldots, M_{K-1}\right\}$ except, perhaps, one of them (with cost, at least, $2(K-2))$ and $F\left(x^{k}\right) \geq 2(K-2)+(K-2)|F|$ holds. Note that, in this case, $2(K-2)+(K-2)|F|$ is the RHS of (11).

If vehicle $k$ services all the edges in $F$ except one of them and all the edges in $H$ then $y^{k}(F)=|F|-1$ and the RHS of the inequality (11) is $2(K-1)+(K-2)(|F|-2)$. In this case, vehicle $k$ must traverse the $|F|-1$ edges it services in $F$ at least once (with cost, at least, $(K-2)(|F|-1))$ and visit the sets $\left\{M_{1}, M_{2}, \ldots, M_{K-1}\right\}$. Given that $|F|-1$ is odd, the 
cheapest way to visit them, with cost $K$, is to begin in $M_{0}$, then $M_{1}, M_{2}$, and so on until $M_{K}$. Hence, $F\left(x^{k}\right) \geq K+(K-2)(|F|-1)=2(K-1)+(K-2)(|F|-2)$.

It is easy to see that the inequality is also satisfied when vehicle $k$ services any other number of edges in $F \cup H$.

Note 3 In order to prove that the above inequalities are facet-inducing, we need to build for each required edge $e \in F \cup H$ a tour for vehicle $k$ servicing all the edges in $F \cup H$ except $e$ and satisfying the inequality as an equality. Obviously if the depot is, for example, in set $M_{1}$, then there is no such tour for the edge $e_{1} \in M_{1}$. Hence, in order to assure that disaggregate inequalities (11) and (12) are facet-inducing we need the depot located in $M_{0} \cup M_{K}$. If the depot $1 \in M_{i}, i \neq 0, K$ then each vehicle $k$ is forced to visit set $M_{i}$ although it does not service the edge $e_{i}$. In this case we define $H=\left\{e_{1}, e_{2}, \ldots, e_{K-1}\right\} \backslash\left\{e_{i}\right\} \subset E_{R}$ and the following disaggregate $\mathrm{K}-\mathrm{C}$ and $\mathrm{K}-\mathrm{C}_{02}$ inequalities

$$
\begin{aligned}
& F\left(x^{k}\right) \geq 2+2 y^{k}(H)+(K-2)\left(2 y^{k}(F)-|F|\right) \\
& F^{\prime}\left(x^{k}\right) \geq 2+2 y^{k}(H)+(K-1)\left(2 y^{k}(F)-|F|\right)
\end{aligned}
$$

are also valid for $\operatorname{KWRPP}(G)$.

Theorem 6 Disaggregate $K-C$ and $K-C_{02}$ inequalities (11) and (12) are facet-inducing for $K W R P P(G)$ if $1 \in M_{0} \cup M_{K}$. Disaggregate $K-C$ and $K-C_{02}$ inequalities (13) and (14) are facet-inducing for $K W R P P(G)$ if $1 \in M_{i}$.

Proof: Again, we will prove the result only for K-C inequalities. Let us suppose $k=1$. Consider the ( 1 vehicle) WRPP instance defined on a graph $G^{\prime}$, based on graph $G$, where the required edges are only those in set $F$ and those in sets $E_{R}\left(M_{i}\right), i=0,1,2, \ldots, K$. If we consider the K-C configuration on graph $G^{\prime}$ defined by the partition $\left\{M_{0}, M_{1}, M_{2}, \ldots, M_{K-1}, M_{K}\right\}$, the standard K-C inequality $F(x) \geq 2(K-1)+(K-2)|F|$ is facet inducing for $\operatorname{WRPP}\left(G^{\prime}\right)$ and there are $m$ linearly independent tours $w_{1}, w_{2}, \ldots, w_{m}$ for the WRPP on $G^{\prime}$ satisfying $F(x)=2(K-1)+(K-2)|F|$. On the other hand, given that $0 \in \operatorname{aff}(\operatorname{WRPP}(G))$, there are $m$ linearly independent tours $z_{1}, z_{2}, \ldots, z_{m}$ for the WRPP on $G$ which traverse all the edges in $E_{R}$ at least once. Hence, the vectors represented in rows (a), (b), (c) and (g) of the matrix shown in Figure 7 are feasible MM $K$-WRPP solutions satisfying the inequality as an equality.

For each $e \in F \cup H$, the vector $w_{i}^{*}$ in rows (d) and (e) represents a WRPP tour on $G^{\prime}$ traversing all the required edges in $F \cup H$ except $e$ and satisfying the inequality as an equality. It is easy to see that we can find such a tour for inequalities (11) (inequalities (13)) when the depot is in $M_{0} \cup M_{K}\left(M_{i}\right)$. For each edge $e \in E_{R} \backslash(F \cup H)$, the vector $w_{i}^{* *}$ in rows (f) represents a tour similar to those in set $\left\{w_{1}, w_{2}, \ldots, w_{m}\right\}$ which traverses all the edges in $F \cup H$ exactly once and edge $e$. It can be seen that the rows of the matrix shown in Figure 7 represent feasible MM $K$-WRPP solutions on $G$ and they are linearly independent.

Note 4 In the definition of the partition $\left\{M_{0}, M_{1}, M_{2}, \ldots, M_{K-1}, M_{K}\right\}$ for disaggregate K-C and $\mathrm{K}_{-} \mathrm{C}_{02}$ inequalities, we do not assume that the required edges are only in set $\left(M_{0}: M_{K}\right)$ and in sets $E\left(M_{i}\right)$. The role of 'required' edges for vehicle $k$ is played by edges in sets $F$ 


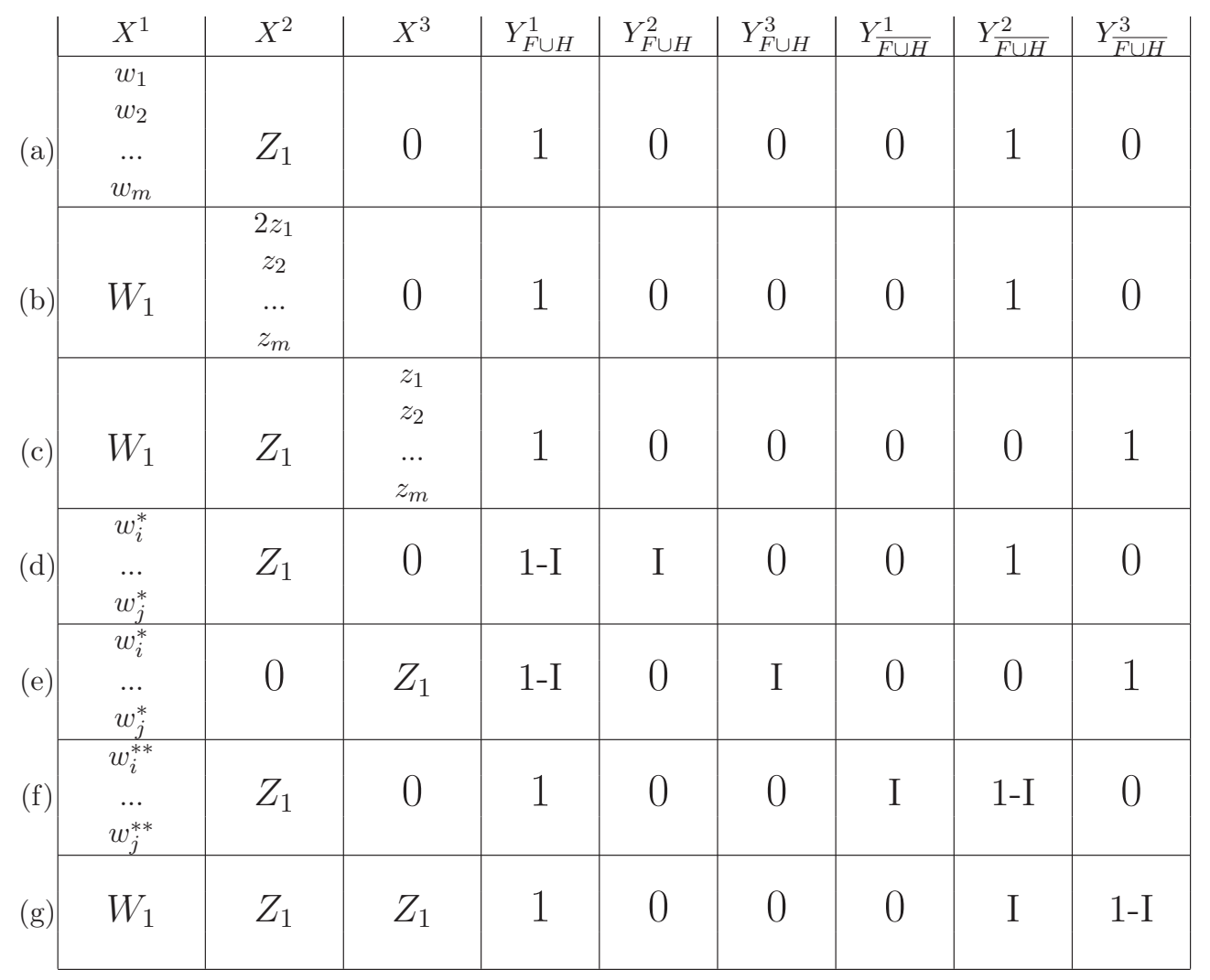

Figure 7: MM $K$-WRPP solutions satisfying inequalities (11) and (13) with equality.

and $H$. Hence, these inequalities also apply for the $\widehat{K}$-vehicles Windy Postman Problem (in which all the edges in $G$ are required).

As with parity inequalities, we can consider aggregate $\mathrm{K}-\mathrm{C}$ inequalities related to a subset of vehicles. If, for example, all the required edges in $F \cup H$ are serviced by a subset of vehicles, then the sum of the routes performed by these vehicles satisfies the standard K-C inequality. Given a partition $\left\{M_{0}, M_{1}, M_{2}, \ldots, M_{K-1}, M_{K}\right\}$ of $V$ and two subsets $F$ and $H$ as above, for each subset $\Omega=\left\{k_{1}, k_{2}, \ldots, k_{P}\right\}$ of $P$ vehicles we will call $\mathrm{P}$-aggregate $\mathrm{K}$-C inequalities to the following ones, where set $H$ is defined as $\left\{e_{1}, e_{2}, \ldots, e_{K-1}\right\}$ such that each $e_{i} \in E\left(M_{i}\right)$ or $\left\{e_{1}, e_{2}, \ldots, e_{K-1}\right\} \backslash\left\{e_{i}\right\}$, depending on wether the depot is located in $M_{0} \cup M_{K}$ or in any other set $M_{i}$ :

$$
\begin{gathered}
\sum_{k \in \Omega} F\left(x^{k}\right) \geq \sum_{k \in \Omega} 2 y^{k}(H)+(K-2)\left(\sum_{k \in \Omega} 2 y^{k}(F)-|F|\right) \\
\sum_{k \in \Omega} F\left(x^{k}\right) \geq 2+\sum_{k \in \Omega} 2 y^{k}(H)+(K-2)\left(\sum_{k \in \Omega} 2 y^{k}(F)-|F|\right)
\end{gathered}
$$

and, similarly, we will call $\mathrm{P}$-aggregate $\mathrm{K}-\mathrm{C}_{02}$ inequalities to

$$
\begin{gathered}
\sum_{k \in \Omega} F^{\prime}\left(x^{k}\right) \geq \sum_{k \in \Omega} 2 y^{k}(H)+(K-1)\left(\sum_{k \in \Omega} 2 y^{k}(F)-|F|\right) \\
\sum_{k \in \Omega} F^{\prime}\left(x^{k}\right) \geq 2+\sum_{k \in \Omega} 2 y^{k}(H)+(K-1)\left(\sum_{k \in \Omega} 2 y^{k}(F)-|F|\right)
\end{gathered}
$$


Theorem 7 -aggregate $K-C$ and $K-C_{02}$ inequalities (15) to (18) are valid for $K W R P P(G)$.

Proof: It is similar to that of Theorem 5 and is omitted here for the sake of brevity.

Theorem 8 -aggregate $K$ - $C$ and $K$ - $C_{02}$ inequalities (15) and (17) (the depot $1 \in M_{0} \cup M_{K}$ ) are facet-inducing for $K W R P P(G)$. P-aggregate $K$-C inequalities (16) are facet-inducing for $K W R P P(G)$ if, and only if, $K \neq 3$ or $|F| \neq 2$. P-aggregate $K-C_{02}$ inequalities (18) are facet-inducing for $K W R P P(G)$ if, and only if, $K \neq 2$ or $|F| \neq 2$.

Proof: Let us prove that P-aggregate $\mathrm{K}-\mathrm{C}$ inequalities are facet inducing. The proof for the $\mathrm{P}$-aggregate $\mathrm{K}-\mathrm{C}_{02}$ inequalities is similar. For the sake of simplicity, let us suppose that $\widehat{K}=3$ and $P=2\left(k_{1}=1, k_{2}=2\right)$. Consider, as in the proof of Theorem 6 , the ( 1 vehicle) WRPP instance defined on a graph $G^{\prime}$, where the required edges are only those in set $F$ and in sets $E_{R}\left(M_{i}\right), i=0,1,2, \ldots, K$. Let $w_{1}, w_{2}, \ldots, w_{m}$ be $m$ linearly independent tours for the WRPP on $G^{\prime}$ satisfying $F(x)=2(K-1)+(K-2)|F|$ and let $z_{1}, z_{2}, \ldots, z_{m}$ be $m$ linearly independent tours for the WRPP on $G$. On the other hand, vectors $w_{i}^{*}$ in rows (d) and (e) for each $e \in F \cup H$, and vectors $w_{i}^{* *}$ in rows (f) and (g) for each edge $e \in E_{R} \backslash(F \cup H)$ are defined as those in Theorem 6 . The vectors represented in the rows of the matrix shown in Figure 8 are feasible MM $K$-WRPP solutions satisfying the inequality as an equality. To prove that this matrix is of full rank, we subtract rows in (e) from rows in (d), obtaining a new row block (d') shown at the bottom of Figure 8 .

Let us assume the depot $1 \in M_{0}$. If we compute $F\left(x^{2}\right)$ on the columns corresponding to the variables labeled $X^{2}$ and divide this sum by $\alpha=F\left(w_{i}\right)=2|H|+(K-2)|F|=$ $2(K-1)+(K-2)|F|$, we obtain a column with all its entries in (b) equal to 1 . By subtracting this column from those corresponding to $Y_{F \cup H}^{2}$ we obtain a 0 block in the rows in (b), and block $\mathrm{B}=\mathrm{I}-1$ in $\left(\mathrm{d}^{\prime}\right)$ is also transformed into a new matrix $B^{*}$, whose columns are those in $B$ minus the column $F\left(-w_{i}^{*}\right) / \alpha$. We proceed as in Theorem 1 to show that $\mathrm{B}^{*}$ is of full rank. To prove this, it suffices to see that matrix $\bar{B}$ shown in Figure 9 is of full rank if, and only if, $K \neq 2$.

If the depot $1 \in M_{i}$, the same process with $\alpha=F\left(w_{i}\right)=2+2|H|+(K-2)|F|=$ $2+2(K-2)+(K-2)|F|$ leads to prove that inequalities (16) are facet-inducing for $\operatorname{KWRPP}(G)$ if, and only if, $K \neq 3$ or $|F| \neq 2$.

Finally, it can be seen that, when $K=3$ and $|F|=2$, P-aggregate K-C inequality (16) can be obtained as the sum of the $\mathrm{P}$ disaggregate $\mathrm{K}-\mathrm{C}$ inequalities (13) associated with the $\mathrm{P}$ vehicles in set $\Omega$. The same result is obtained for $\mathrm{P}$-aggregate $\mathrm{K}-\mathrm{C}_{02}$ inequalities (18) when $K=|F|=2$.

\subsection{Honeycomb and Honeycomb $b_{02}$ inequalities}

Honeycomb (HC) inequalities are known to be valid and facet-inducing inequalities for the WGRP ([10]) and they are a generalization of K-C inequalities. In a K-C configuration, a $R$-set (or a cluster of $R$-sets) is divided into two parts $\left(M_{0}\right.$ and $\left.M_{K}\right)$. The Honeycomb inequalities generalize this configuration simultaneously both in the number of parts a $R$-set is divided into and in the number of $R$-sets we divide. Nevertheless, we restrict ourselves here to Honeycomb configurations in which only one $R$-set (or cluster of $R$-sets) is divided 


\begin{tabular}{|c|c|c|c|c|c|c|c|c|}
\hline$X^{1}$ & $X^{2}$ & $X^{3}$ & $Y_{F \cup H}^{1}$ & $Y_{F \cup H}^{2}$ & $Y_{F \cup H}^{3}$ & $Y_{F \cup H}^{1}$ & $Y_{F \cup H}^{2}$ & $Y \frac{3}{F \cup H}$ \\
\hline (a) $\begin{array}{c}w_{1} \\
w_{2} \\
\ldots \\
w_{m} \\
\end{array}$ & 0 & $Z_{1}$ & 1 & 0 & 0 & 0 & 0 & 1 \\
\hline (b) & $\begin{array}{c}w_{1} \\
w_{2} \\
\ldots \\
w_{m} \\
\end{array}$ & $Z_{1}$ & 0 & 1 & 0 & 0 & 0 & 1 \\
\hline$W_{1}$ & 0 & $\begin{array}{c}2 z_{1} \\
z_{2} \\
\ldots \\
z_{m} \\
\end{array}$ & 1 & 0 & 0 & 0 & 0 & 1 \\
\hline $\begin{array}{c}w_{i}^{*} \\
\ldots \\
w_{j}^{*}\end{array}$ & 0 & $Z_{1}$ & $1-\mathrm{I}$ & 0 & I & 0 & 0 & 1 \\
\hline (e) & $\begin{array}{c}w_{i}^{*} \\
\ldots \\
w_{j}^{*}\end{array}$ & $Z_{1}$ & 0 & $1-\mathrm{I}$ & I & 0 & 0 & 1 \\
\hline \begin{tabular}{l|c} 
& $w_{i}^{* *}$ \\
$\ldots$ \\
$\ldots$ \\
$w_{j}^{* *}$
\end{tabular} & 0 & $Z_{1}$ & 1 & 0 & 0 & I & 0 & $1-\mathrm{I}$ \\
\hline (g) & $\begin{array}{c}w_{i}^{* *} \\
\ldots \\
w_{j}^{* *} \\
\end{array}$ & $Z_{1}$ & 0 & 1 & 0 & 0 & I & $1-\mathrm{I}$ \\
\hline $\begin{array}{c}w_{i}^{*} \\
\ldots \\
w_{j}^{*}\end{array}$ & $\begin{array}{c}-w_{i}^{*} \\
\ldots \\
-w_{j}^{*}\end{array}$ & 0 & $1-\mathrm{I}$ & I-1 & 0 & 0 & 0 & 0 \\
\hline
\end{tabular}

Figure 8: MM $K$-WRPP solutions satisfying (15) as an equality.

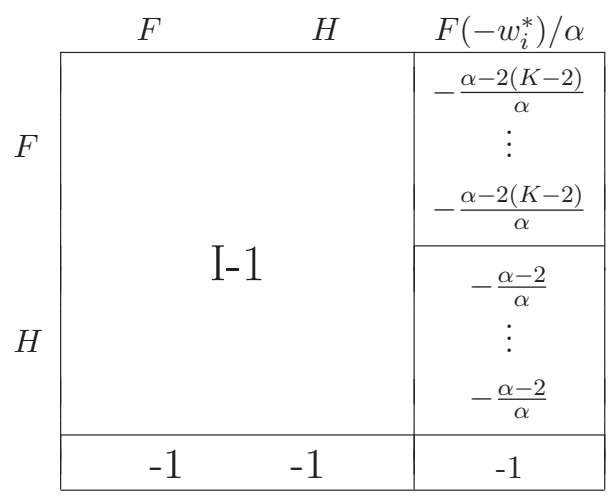

Figure 9: Matrix $\bar{B}$ of Theorem 8 .

into several parts. They are defined by

- a partition $\left\{W_{1}, \ldots, W_{L}, M_{1}, \ldots, M_{K-1}\right\}$ of $V$, with $L \geq 2, K \geq 3$, such that $\left(W_{1} \cup \ldots \cup W_{L}\right), M_{1}, \ldots, M_{K-1}$ are clusters of one or more $R$-sets, $\delta\left(W_{i}\right)$ contains a positive and even number of required edges for all $i$ and the graph induced by the required edges on the vertex set $\left\{W_{1}, \ldots, W_{L}\right\}$ is connected.

- a tree $T$ spanning the sets $W_{1}, \ldots, W_{L}, M_{1}, \ldots, M_{K-1}$ such that the degree in $T$ of 
every vertex set $W_{i}$ is 1 , the degree of vertex sets $M_{j}$ is at least 2 and the number of edges of the unique path in the tree connecting any distinct $W_{i}, W_{j}, d\left(W_{i}, W_{j}\right)$, is 3 or more.

Note that when $L=2$ the tree $T$ is a path and we have a K-C configuration. If $L \geq 3$, then $K \geq 4$ is needed for $d\left(W_{i}, W_{j}\right)$ to be 3 or more. The costs of such a HC configuration are $c\left(M_{i}, M_{j}\right)=d\left(M_{i}, M_{j}\right), c\left(M_{i}, W_{j}\right)=d\left(M_{i}, W_{j}\right)$ and $c\left(W_{i}, W_{j}\right)=d\left(W_{i}, W_{j}\right)-2$. The corresponding Honeycomb inequality is then

$$
F(x)=\sum_{(i, j) \in E}\left(\alpha_{i j} x_{i j}+\alpha_{j i} x_{j i}\right) \geq 2(K-1)+\sum_{(i, j) \in \mathcal{E}_{R}} \alpha_{i j}
$$

where $\alpha_{i j}=c\left(M_{p}, M_{q}\right)$ if $i \in M_{p}$ and $j \in M_{q}\left(\alpha_{i j}=c\left(M_{p}, W_{q}\right)\right.$ if $i \in M_{p}$ and $j \in W_{q}$ and so on $)$ and $\mathcal{E}_{R}$ is the set of required edges joining two distinct sets $W_{i}, W_{j}$. Note that, for edges in $\mathcal{E}_{\mathcal{R}}, \alpha_{i j}=\alpha_{j i}$, and therefore we use only $\alpha_{i j}$ in the RHS of the inequality. Figure 10(a) shows a Honeycomb configuration. The bold lines represent the required edges and the thin lines represent edges in the spanning tree $T$. The RHS for this example is $2(8-1)+(1+4+1+6)$.

Honeycomb $02\left(\mathrm{HC}_{02}\right)$ inequalities are associated with a similar configuration (see figure $10(\mathrm{~b})$ ). In this case, nodes $W_{i}$ are classified into two types, nodes of type $\mathcal{O}$ (those that are incident with edges in $T$ with coefficients 0 and 2) and nodes of type $\mathcal{I}$, in such a way that there is at least one node of each type. We will assume that $d\left(W_{i}, W_{j}\right) \geq 3$ if $W_{i}$ and $W_{j}$ are nodes of the same type, while $d\left(W_{i}, W_{j}\right) \geq 2$ otherwise. The costs are now $c\left(W_{i}, W_{j}\right)=d\left(W_{i}, W_{j}\right)-2$ if $W_{i}$ and $W_{j}$ are nodes of the same type, $c\left(W_{i}, W_{j}\right)=d\left(W_{i}, W_{j}\right)-1$ if $W_{i}$ and $W_{j}$ are nodes of different types, $c\left(W_{i}, M_{q}\right)=0$ and $c\left(M_{q}, W_{i}\right)=2$ if $W_{i}$ is of type $\mathcal{O}$ and $\left(W_{i}, M_{q}\right) \in T$, the other edges in $T$ have cost 1 and for all the remaining edges the cost is the shortest path cost in $T$. With these coefficients, the $\mathrm{HC}_{02}$ inequality is expressed as in (19). The RHS for the inequality in Figure 10(b) is $2(8-1)+(1+5+2+6)$.

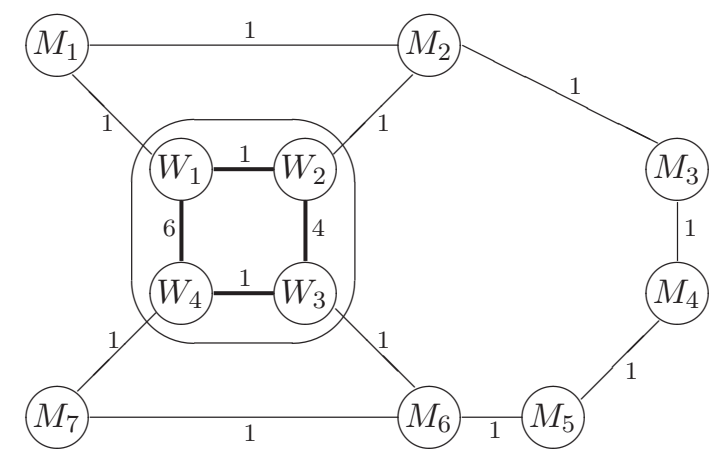

(a)

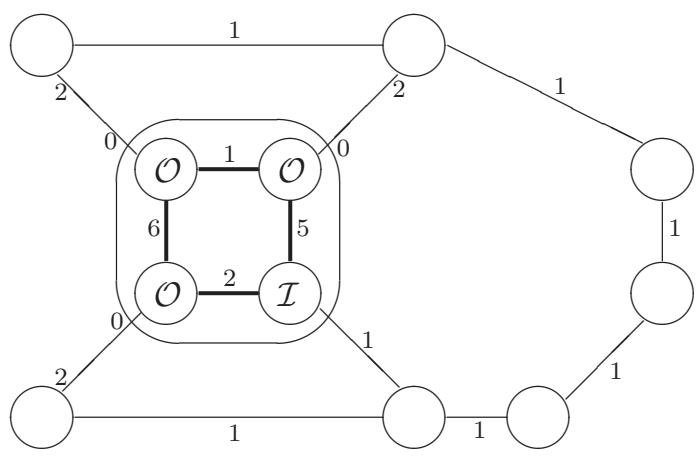

(b)

Figure 10: Honeycomb and Honeycomb 02 Configurations

Let $F(x) \geq 2(K-1)+\alpha_{R}$ be a $\mathrm{HC}$ or a $\mathrm{HC}_{02}$ inequality associated with a configuration as above, where $\alpha_{R}=\sum_{(i, j) \in \mathcal{E}_{R}} \alpha_{i j}$. The corresponding aggregate $\mathrm{HC}$ or $\mathrm{HC}_{02}$ inequality is then

$$
\sum_{k=1}^{\widehat{K}} F\left(x^{k}\right) \geq 2(K-1)+\alpha_{R},
$$


and, as it has been mentioned in Section 2, is valid for the MM $K$-WRPP.

Just like for $\mathrm{KC}$ inequalities, we describe in what follows the disaggregate version of the $\mathrm{HC}$ inequalities. Consider a partition $\left\{W_{1}, \ldots, W_{L}, M_{1}, \ldots, M_{K-1}\right\}$ of $V$ (where subgraphs $\mathrm{G}\left(W_{i}\right), \mathrm{G}\left(M_{j}\right)$ are connected) and a tree $T$ spanning the sets $W_{1}, \ldots, W_{L}, M_{1}, \ldots, M_{K-1}$ and satisfying the same degree conditions described above. Let $F \subset \mathcal{E}_{R}$ be such that each subset $\delta\left(W_{i}\right) \cap F$ contains a positive and even number of edges and the graph induced by edges in $F$ on the vertex set $\left\{W_{1}, \ldots, W_{L}\right\}$ is connected. Let $H=\left\{e_{1}, e_{2}, \ldots, e_{K-1}\right\} \subset E_{R}$ be a subset of edges such that each $e_{i} \in E\left(M_{i}\right)$. For each vehicle $k$ we will call disaggregate $\mathrm{HC}$ and $\mathrm{HC}_{02}$ inequalities to:

$$
F\left(x^{k}\right) \geq 2 y^{k}(H)+\sum_{e=(i, j) \in F} \alpha_{i j}\left(2 y_{e}^{k}-1\right)
$$

Theorem 9 Disaggregate $H C$ and $H C_{02}$ inequalities (21) are valid for $K W R P P(G)$.

Proof: We will prove the theorem for disaggregate HC inequalities. The proof for disaggregate $\mathrm{HC}_{02}$ is similar. If vehicle $k$ services all the edges in $F \cup H$, then $y_{e}^{k}=1$ for all $e \in F \cup H$ and the RHS of inequality (21) is $2(K-1)+\alpha_{R}^{\prime}$, where $\alpha_{R}^{\prime}=\sum_{(i, j) \in F} \alpha_{i j}$. In this case, vehicle $k$ must traverse the edges in $F$, with cost $\alpha_{R}^{\prime}$, and visit all the sets $M_{i}$, with cost at least $2(K-1)$, therefore $F\left(x^{k}\right) \geq 2(K-1)+\alpha_{R}^{\prime}$ holds.

If vehicle $k$ services all the edges in $F \cup H$ except one of them in $H$, then vehicle $k$ must traverse the edges in $F$, with $\operatorname{cost} \alpha_{R}^{\prime}$, and visit the sets $M_{i}$ except, perhaps, one of them, with cost at least $2(K-2)$. Then $F\left(x^{k}\right) \geq 2(K-2)+\alpha_{R}^{\prime}$, which, in this case, is the RHS of (21).

If vehicle $k$ services all the edges in $F \cup H$ except one of them, say $e_{r s}$, in $F$, then the RHS of inequality (21) is $2(K-1)+\alpha_{R}^{\prime}-2 \alpha_{r s}$. In this case, vehicle $k$ must traverse the remaining edges in $F$ at least once, with cost $\alpha_{R}^{\prime}-\alpha_{r s}$, and visit all the sets $M_{i}$. Given that sets $W_{r}, W_{s}$ are incident with an odd number of edges in $F \backslash\left\{e_{r s}\right\}$, the cheapest way to do this is to traverse the path in $T$ connecting $W_{r}$ and $W_{s}$, with cost $\alpha_{r s}+2$, and visit the remaining $K-\alpha_{r s}-2$ sets $M_{i}$ using edges in $T$ twice. Hence, $F\left(x^{k}\right) \geq \alpha_{R}^{\prime}-\alpha_{r s}+\left(\alpha_{r s}+2\right)+2\left(K-\alpha_{r s}-2\right)=$ $2(K-1)+\alpha_{R}^{\prime}-2 \alpha_{r s}$.

It is easy to see that the inequality is also satisfied when vehicle $k$ services any other number of edges in $F \cup H$.

Like for disaggregate $\mathrm{K}-\mathrm{C}$ inequalities, if the depot is in a set $M_{i}$, for example $1 \in M_{1}$, we define $H=\left\{e_{2}, e_{3}, \ldots, e_{K-1}\right\}$ and the disaggregate $\mathrm{HC}$ and $\mathrm{HC}_{02}$ inequalities are

$$
F\left(x^{k}\right) \geq 2+2 y^{k}(H)+\sum_{e=(i, j) \in F} \alpha_{i j}\left(2 y_{e}^{k}-1\right)
$$

It is easy to see that inequalities (22) are valid for the MM K-WRPP.

Finally, for each subset $\Omega=\left\{k_{1}, k_{2}, \ldots, k_{P}\right\}$ of $P$ vehicles we will call P-aggregate HC inequalities to the following ones, where set $H$ is defined as $\left\{e_{1}, e_{2}, \ldots, e_{K-1}\right\}$ such that each $e_{i} \in E\left(M_{i}\right)$ or $\left\{e_{1}, e_{2}, \ldots, e_{K-1}\right\} \backslash\left\{e_{i}\right\}$, depending on wether the depot is located in a set $W_{j}$ or in a set $M_{i}$ :

$$
\sum_{k \in \Omega} F\left(x^{k}\right) \geq \sum_{k \in \Omega} 2 y^{k}(H)+\sum_{e=(i, j) \in F} \alpha_{i j}\left(\sum_{k \in \Omega} 2 y_{e}^{k}-1\right)
$$




$$
\sum_{k \in \Omega} F\left(x^{k}\right) \geq 2+\sum_{k \in \Omega} 2 y^{k}(H)+\sum_{e=(i, j) \in F} \alpha_{i j}\left(\sum_{k \in \Omega} 2 y_{e}^{k}-1\right)
$$

Similarly, we can define P-aggregate $\mathrm{HC}_{02}$ inequalities depending on the location of the depot. Their general expression is exactly the same as (23) and (24), being the only difference the definition of coefficients $\alpha_{i j}$.

Theorem 10 P-aggregate $H C$ (and $H_{02}$ ) inequalities (23) and (24) are valid for $K W R P P(G)$.

Proof: It is similar to that of Theorem 9 and is omitted here for the sake of brevity.

\section{Max-length constraints}

In Benavent et al. [7], a heuristic algorithm for the MM $K$-WRPP is proposed that provides quite tight upper bounds. In this section we describe how we use these upper bounds for introducing inequalities that are satisfied by any optimal solution. However, these inequalities are not valid for the MM $K$-WRPP because they may not be satisfied by some feasible solutions. Let $u b$ be a known upper bound for a given MM $K$-WRPP instance. Given a set of required edges $F$, let $z(W G R P(F))$ be the optimal cost of the Windy General Routing Problem instance defined on the graph $\mathrm{G}$, considering the depot as the only required vertex and $F$ as the set of required edges. Let us denote $n v(F)=\lceil z(W G R P(F)) / u b\rceil$, then, in every optimal MM $K$-WRPP solution, at least $n v(F)$ vehicles will be needed to serve all the edges in $F$ so a single vehicle will serve at most $p-n v(F)+1$ of these edges, and the inequalities

$$
\sum_{e \in F} y_{e}^{k} \leq p-n v(F)+1, \quad \forall \text { vehicle } k
$$

have to be satisfied by any optimal solution. We call them disaggregate max-length inequalities associated with set $F$.

Now, given any set of vertices $S \subset V \backslash\{1\}$, let us consider the set of edges $F_{S}=E_{R}(S) \cup$ $\delta_{R}(S)$. Then, at least $n v\left(F_{S}\right)$ vehicles will cross the edge cutset $\delta(S)$ in any optimal solution. Therefore, the optimal solutions of the MM $K$-WRPP will satisfy the following aggregate max-length inequality:

$$
\sum_{k=1}^{\widehat{K}} x^{k}(\delta(S)) \geq 2 n v\left(F_{S}\right)
$$

These inequalities can be generalized by considering subsets of vehicles instead of all of them. Thus, if $\Omega=\left\{k_{1}, k_{2}, \ldots, k_{P}\right\}$ is a subset of $P$ vehicles we will call P-aggregate max-length inequalities to:

$$
\sum_{k \in \Omega} x^{k}(\delta(S)) \geq 2\left(n v\left(F_{S}\right)-(\widehat{K}-P)\right)
$$

Inequalities (27) must be satisfied by any optimal solution because, even if all the vehicles not in $\Omega$ cross the edge cutset $\delta(S)$, at least $n v\left(F_{S}\right)-(\widehat{K}-P)$ vehicles in the subset $\Omega$ will be needed to service all the edges in $F_{S}$. 


\section{The Branch-and-Cut algorithm}

In this section we present an enhanced branch-and-cut algorithm, based on the procedure for the MM $K$-WRPP described in [6]. This new version incorporates separation algorithms for the inequalities described in this paper as well as an upper bound obtained by the metaheuristic algorithm presented in Benavent et al. [7].

\subsection{Initial relaxation}

The initial LP relaxation contains equations (2), assuring that each required edge is serviced by exactly one vehicle, symmetry equations (4), min-max inequalities (1), traversing inequalities (3), an inequality for each vehicle forcing it to leave the depot, and a set of constraints proposed in [13] that avoid symmetric solutions (see [6]). The first of these anti-symmetry constraints assigns a required edge to the first vehicle. In [6], this edge is chosen as the one farthest from the depot using an average distance. Now, as suggested by Desaulniers [11], we choose the edge $e=(i, j)$ as the one that maximizes $\min \left\{l_{1 i}+c_{i j}+l_{j 1}, l_{1 j}+c_{j i}+l_{i 1}\right\}$, where $l_{u v}$ is the cost of the shortest path from vertex $u$ to vertex $v$. By means of this assignment, we guarantee that the lower bound at the end of the root node will be greater or equal than the cost of any route servicing only one required edge.

Finally, several aggregate max-length constraints (26) are added. Let us denote by $C_{1}$ the connected component in graph $G_{R}$ containing the depot. For each connected component $C_{i}, i \neq 1$, we define $F=E_{R}\left(C_{i}\right)$. If $n v(F)>1$, we add the aggregate max-length inequality associated with $F$. Moreover, we consider sets formed by more than one connected component. Given any $C_{i}$, its farthest adjacent connected component $C_{j}, j \neq 1$, is selected, and the aggregate max-length inequality associated with $F=E_{R}\left(C_{i}\right) \cup E_{R}\left(C_{j}\right)$ is added if $n v(F)>1$. We continue enlarging $F$ in this way until only $C_{1}$ is left to add.

\subsection{Separation Algorithms}

In this section, we present the separation algorithms that have been used to identify the following types of inequalities that are violated by the current LP solution at any iteration of the cutting plane algorithm: $\mathrm{P}$-aggregate parity inequalities, disaggregate and $\mathrm{P}$-aggregate $\mathrm{K}-\mathrm{C}$ and $\mathrm{K}-\mathrm{C}_{02}$ inequalities and disaggregate, $\mathrm{P}$-aggregate and aggregate max-length inequalities. The separation algorithm for aggregate K-C lifted inequalities is the same as for aggregate $\mathrm{K}-\mathrm{C}$ with minor changes. Separation procedures for the other types of inequalities were presented in [6].

Given an LP solution $\left(\bar{x}^{1}, \bar{y}^{1}, \bar{x}^{2}, \bar{y}^{2}, \ldots, \bar{x}^{\widehat{K}}, \bar{y}^{\widehat{K}}\right) \in \mathbb{R}^{\left(2|E|+\left|E_{R}\right|\right) \widehat{K}}$ we define the P-aggregate vector $\bar{x}^{\Omega}$ associated with a subset $\Omega=\left\{k_{1}, k_{2}, \ldots, k_{P}\right\}$ of $\mathrm{P}$ vehicles as $\bar{x}^{\Omega}=\sum_{k \in \Omega} \bar{x}^{k}$ and its associated weighted graph $\bar{G}^{\Omega}=\left(\bar{V}^{\Omega}, \bar{E}^{\Omega}, \bar{x}^{\Omega}, \bar{y}^{\Omega}\right)$, where $\bar{V}^{\Omega}, \bar{E}^{\Omega}$ are the sets of vertices and edges of the subgraph of $G$ induced by the edges $e \in E$ such that $\bar{x}_{e}^{\Omega}=\bar{x}_{i j}^{\Omega}+\bar{x}_{j i}^{\Omega}>0$, plus the depot node, if necessary.

Note that in the case $P=\widehat{K}$, i.e. $\Omega=\{1,2, \ldots, \widehat{K}\}, \bar{G}^{\Omega}$ is the 'aggregate' graph used as input to the separation procedures described in Corberán et al. [6] to find aggregate connectivity, $R$-odd cut, $\mathrm{KC}, \mathrm{KC}_{02}, \mathrm{HC}$ and $\mathrm{HC}_{02}$ inequalities violated by $\left(\bar{x}^{1}, \bar{y}^{1}, \bar{x}^{2}, \bar{y}^{2}, \ldots, \bar{x}^{\widehat{K}}, \bar{y}^{\widehat{K}}\right)$. 
The graphs $\bar{G}^{\Omega}$ corresponding to all the other cases, $\Omega=\left\{k_{1}, k_{2}, \ldots, k_{P}\right\}$ with $P<\widehat{K}$, are the graphs used for the separation of the disaggregate $(P=1)$ and P-aggregate $(1<P<\widehat{K})$ inequalities, respectively. We want to point out that both disaggregate and $\mathrm{P}$-aggregate inequalities of a given family can be separated with the same procedure changing only the corresponding input graph $\bar{G}^{\Omega}$.

The separation of P-aggregate parity inequalities is, therefore, done as described in [6] for the disaggregate parity inequalities.

\subsubsection{Separation of disaggregate and $\mathrm{P}$-aggregate $\mathrm{K}-\mathrm{C}$ and $\mathrm{K}-\mathrm{C}_{02}$ inequalities}

In this section we describe the separation of disaggregate $\mathrm{KC}$ and $\mathrm{K}-\mathrm{C}_{02}$ inequalities (11) to (14) and $\mathrm{P}$-aggregate $\mathrm{KC}$ and $\mathrm{K}-\mathrm{C}_{02}$ inequalities (15) to (18).

Given an LP solution $\left(\bar{x}^{1}, \bar{y}^{1}, \bar{x}^{2}, \bar{y}^{2}, \ldots, \bar{x}^{K}, \bar{y}^{K}\right)$ and a subset $\Omega$ of $P<\widehat{K}$ vehicles, we build the corresponding support graph $\bar{G}^{\Omega}$ and label as 'required' the depot and the edges $e \in E_{R}$ such that $\bar{y}_{e}^{\Omega}>1-\epsilon_{y}$, where $\epsilon_{y}$ is a given parameter. Then, we apply the heuristic algorithm described in Plana [15] for the WGRP (based on the one presented in [8] for the undirected GRP) to obtain the sets $M_{0}, M_{1}, M_{2}, \ldots, M_{K}$. Set $F$ is formed by the edges in $M_{0} \cup M_{K}$ labeled 'required'. If the depot $1 \in M_{0} \cup M_{K}$, we select for each set $M_{j}$, $j=1, \ldots, K-1$, the edge $e_{j} \in E\left(M_{j}\right)$ that maximizes $\bar{y}_{e_{j}}^{\Omega}$. We define $H=\left\{e_{1}, e_{2}, \ldots, e_{K-1}\right\}$ and check the corresponding inequality (11), (12), (15) or (17) for violation. On the other hand, if the depot $1 \in M_{i}, i \neq 0, i \neq K$, we define the set $H$ as above except for edge $e_{i}$, and check the corresponding inequality (13), (14), (16) or (18) for violation. If no violated inequality is found, we try to shrink the $\mathrm{KC}$ configuration by merging some sets $M_{j}$ as in the heuristic algorithm described in [15].

Several values for $\epsilon_{y}$ have been tried. After some computational testing with values 0.2 , 0.4 and 0.65 , we decided to finally set $\epsilon_{y}=0.2$.

\subsubsection{Separation of max-length inequalities}

Recall that max-length inequalities are interesting only when $n v(F)>1$ for a set of required edges $F$, and computing $n v(F)$ involves solving exactly a WGRP. In order to avoid duplicate computations, we maintain two lists of sets of required edges, denoted $L_{1}$ and $L_{2}$. List $L_{1}$ includes the sets $F$ of required edges for which $n v(F)>1$, while $L_{2}$ includes those for which $n v(F)=1$. Initially $L_{1}=L_{2}=\emptyset$ and the lists are updated each time $n v(F)$ is computed for a given set $F$.

Disaggregate max-length inequalities (25) are separated as follows. Given an LP solution $\left(\bar{x}^{1}, \bar{y}^{1}, \bar{x}^{2}, \bar{y}^{2}, \ldots, \bar{x}^{\widehat{K}}, \bar{y}^{\widehat{K}}\right)$, for each vehicle $k, k=1, \ldots, \widehat{K}$, let $\left\{e_{1}, e_{2}, \ldots, e_{q}\right\} \subset E_{R}$ be an ordered set of required edges such that $\bar{y}_{e_{j}}^{k}>0.9$ and $\bar{y}_{e_{1}}^{k} \geq \bar{y}_{e_{2}}^{k} \geq \ldots, \geq \bar{y}_{e_{q}}^{k}$. Then, let $F=\left\{e_{1}, e_{2}, \ldots, e_{f}\right\}$ where, $f$ is the maximal number such that $\sum_{e \in F} \bar{y}_{e}^{k}>|F|-1+\epsilon$ (we set $\epsilon=0.5$, initially). Iteratively, for each required edge $\bar{e}$ with $0.5<\bar{y}_{\bar{e}}^{k}<0.9$, we consider the set $\bar{F}=F \cup\{\bar{e}\}$. Now, lists $L_{1}$ and $L_{2}$ are scanned looking for sets related to $\bar{F}$. Three cases are possible: (a) there is a set $F^{\prime} \in L_{1}$ such that $\bar{F} \supseteq F^{\prime}$, in which case we know that $n v(\bar{F})>1$, (b) there is a set $F^{\prime} \in L_{2}$ such that $\bar{F} \subseteq F^{\prime}$, so $n v(\bar{F})=1$, and (c) neither (a) nor (b) holds and $n v(\bar{F})$ is computed solving the WGRP associated with $\bar{F}$. In the case (a), 
if $\bar{F} \supset F^{\prime}$, an estimation of the real value $n v(\bar{F})$ is made and if it is found likely that $n v(\bar{F})$ is greater than $n v\left(F^{\prime}\right), n v(\bar{F})$ is exactly computed solving the associated WGRP, otherwise, $n v(\bar{F})=n v\left(F^{\prime}\right)$ is assumed. Finally, we set $\epsilon=0$ and repeat the process for finding $F$ again, but without adding last edge $\bar{e}$.

In the case that a subset $F$ is found for which the corresponding inequality (25) is violated, then we also check if an aggregate max-length inequality (26) is violated for the set $S^{\prime}$ of vertices incident with the edges in $F$.

Aggregate max-length inequalities (26) are also separated using the following heuristic. Starting with vertex selected at random (in such a way that each vertex has a probability of being chosen proportional to its distance to the depot), we build a sequence of vertex subsets by adding each time a new vertex in such a way that for the resulting subset $S, \sum_{k=1}^{\widehat{K}} \bar{x}^{k}(\delta(S))$ is minimum. For each generated subset, the set of edges $F_{S}=E_{R}(S) \cup \delta_{R}(S)$ is built and lists $L_{1}$ and $L_{2}$ are used as before to estimate the value of $n v\left(F_{S}\right)$. If the corresponding inequality (26) is violated, it is added to the LP. Furthermore, if $n v\left(F_{S}\right)>1$ holds, then the corresponding P-aggregate max-length inequality (27) is also checked for violation for the subset of vehicles $\Omega$ for which $\bar{x}^{k}(\delta(S))<2$.

\subsection{The cutting plane algorithm}

At each iteration of the cutting plane algorithm the separation procedures are called in a specific order and the violated inequalities found are added to the LP relaxation. The separation algorithms for aggregate inequalities are called in a similar way as described in [6]:

1. R-odd cut and connectivity separation heuristics.

2. Exact connectivity separation if the corresponding heuristics have failed.

3. Exact R-odd cut separation if no violated inequalities have been found by the corresponding heuristics.

4. Heuristic algorithms for separating $\mathrm{K}-\mathrm{C}, \mathrm{K}-\mathrm{C}$ lifted and $\mathrm{K}-\mathrm{C}_{02}$ inequalities if no violated connectivity inequalities have been found.

5. If the total number of violated inequalities found is less than 20 and no violated connectivity inequalities have been found, run heuristic algorithms for separating $\mathrm{HC}$ and $\mathrm{HC}_{02}$ inequalities.

The separation algorithms for disaggregate and P-aggregate inequalities have been implemented as follows. First, for each vehicle $k$, we use the heuristic and, if necessary, the exact separation procedures for disaggregate connectivity and parity inequalities. Heuristics for identifying violated disaggregate $\mathrm{K}-\mathrm{C}$ and $\mathrm{K}-\mathrm{C}_{02}$ inequalities are applied only when no violated connectivity inequalities are found, since these algorithms assume the corresponding support graph to be connected.

In what refers to the separation of P-aggregate inequalities, we do not know which is the best way to choose the set of vehicles to be aggregated. Given that applying the separation procedures for $\mathrm{P}$-aggregate inequalities for each possible combination of vehicles is computationally expensive, we proceed as follows. 
For any pair of vehicles $\left\{k_{1}, k_{2}\right\}$, we apply the separation procedures for P-aggregate parity inequalities only when no violated disaggregate parity inequality has been found neither for $k_{1}$ nor $k_{2}$. Moreover, for any subset of 3 vehicles (if $\widehat{K} \geq 4$ ) we apply the separation procedures for 3-aggregate parity inequalities only when no violated disaggregate nor 2-aggregate parity inequality associated with any of the 3 vehicles has been found. We proceed in a similar way for subsets of 4 vehicles (if $\widehat{K} \geq 5$ ). The same strategy is used for separating P-aggregate $\mathrm{K}-\mathrm{C}$ and $\mathrm{K}-\mathrm{C}_{02}$ inequalities, with the additional condition that, for a given set $\Omega$ of vehicles, the corresponding support graphs $\bar{G}^{k}, k \in \Omega$, must be connected.

Finally, the separation algorithms for the aggregate, disaggregate and P-aggregate maxlength inequalities described in Section 5.2.2 are executed.

The above cutting plane procedure is applied at each node of the search tree until no new violated inequalities are found. When this happens we branch using the Strong Branching strategy [4] implemented in Cplex (see [6]).

\section{Computational results}

We present here the computational results obtained on the same set of instances used in [6] that can be found in http://www.uv.es/corberan. The B\&C procedure has been coded in $\mathrm{C} / \mathrm{C}++$ using the Cplex 9.0 MIP Solver with Concert Technology 2.0. All the tests were run on an Intel Core 2 at $2.40 \mathrm{GHz}$ with $2 \mathrm{~GB}$ RAM with a time limit of 1 hour.

A MM $K$-WRPP instance consists of a WRPP instance and a given number of vehicles. The characteristics of the 144 WRPP instances used are shown in Table 1 grouped into 24 subsets of 6 instances each, sharing the same underlying graph but having different costs. These instances have up to 50 vertices, 184 edges, 78 required edges and $8 R$-sets. For each WRPP instance, 4 MM $K$-WRPP instances with $2,3,4$ and 5 vehicles have been tested.

Tables 2 to 5 show the computational results obtained by the algorithm proposed in [6] and the enhanced branch-and-cut algorithm presented here for instances with 2 to 5 vehicles, respectively. First column shows the name of the set of instances. Columns 2, 3 and 4 show the number of optima, the average gap at the root node and the average final gap obtained by the branch-and-cut procedure described in [6] with a time limit of 30 minutes. Columns 5,6 and 7 show the corresponding results for the new branch-and-cut, and columns 8 and 9 report the average CPU time in seconds and the average number of nodes of the search tree. The average final gap has been computed only for the instances that were not optimally solved within the specified computing time, while the average time and number of nodes correspond to those that have been solved to optimality. Last row shows, for both algorithms, the total number of solved instances, the average gap at the root node for all the 144 instances and the average final gap for those instances that could not be solved in the specified time limit. All the gaps have been computed with respect to the cost of the best known solution at the time of the experiments and are expressed in percentages.

We consider that, in general, the results are very good. We have been able to solve to optimality all the 144 instances with 2 vehicles, 130 instances with 3 vehicles, 124 with 4 vehicles and 113 with 5 vehicles within a CPU time limit of 1 hour. Overall, a big advance has been achieved when compared with the results presented in [6]. Such improvement is mainly due to the incorporation of separation procedures for the new inequalities presented 


\begin{tabular}{|c|c|c|c|c|}
\hline & $|V|$ & $|E|$ & $\left|E_{R}\right|$ & R-sets \\
\hline $\mathrm{C} 01$ & 11 & 13 & 7 & 4 \\
\hline $\mathrm{C} 02$ & 14 & 33 & 12 & 4 \\
\hline $\mathrm{C} 03$ & 28 & 57 & 26 & 4 \\
\hline $\mathrm{C} 04$ & 17 & 35 & 22 & 3 \\
\hline $\mathrm{C} 05$ & 20 & 35 & 16 & 5 \\
\hline $\mathrm{C} 06$ & 24 & 46 & 20 & 7 \\
\hline $\mathrm{C} 07$ & 23 & 47 & 24 & 3 \\
\hline $\mathrm{C} 08$ & 17 & 40 & 24 & 2 \\
\hline C09 & 14 & 26 & 14 & 3 \\
\hline $\mathrm{C} 10$ & 12 & 20 & 10 & 4 \\
\hline C11 & 9 & 14 & 7 & 3 \\
\hline $\mathrm{C} 12$ & 7 & 18 & 5 & 3 \\
\hline $\mathrm{C} 13$ & 7 & 10 & 4 & 3 \\
\hline C14 & 28 & 79 & 31 & 6 \\
\hline $\mathrm{C} 15$ & 26 & 37 & 19 & 8 \\
\hline C16 & 31 & 94 & 34 & 7 \\
\hline $\mathrm{C} 17$ & 19 & 44 & 17 & 5 \\
\hline C18 & 23 & 37 & 16 & 8 \\
\hline C19 & 33 & 54 & 29 & 7 \\
\hline $\mathrm{C} 20$ & 50 & 98 & 63 & 7 \\
\hline $\mathrm{C} 21$ & 49 & 110 & 67 & 6 \\
\hline $\mathrm{C} 22$ & 50 & 184 & 74 & 6 \\
\hline $\mathrm{C} 23$ & 50 & 158 & 78 & 6 \\
\hline $\mathrm{C} 24$ & 41 & 125 & 55 & 7 \\
\hline Global & 25.13 & 58.92 & 28.08 & 5.04 \\
\hline
\end{tabular}

Table 1: Instance characteristics

here: aggregate $\mathrm{K}-\mathrm{C}$ lifted, disaggregate $\mathrm{K}-\mathrm{C}$ and $\mathrm{K}-\mathrm{C}_{02}$, $\mathrm{P}$-aggregate parity, $\mathrm{K}-\mathrm{C}$ and $\mathrm{K}-\mathrm{C}_{02}$, and max-length inequalities. This is quite apparent if we compare the average gaps at the root node obtained with both procedures. On the other hand, the improvement achieved in the final results is also due to the use of a very tight upper bound provided by the heuristic algorithm described in [7].

With 2 vehicles, all the small size instances have been solved in a few seconds and we have also solved all the 30 medium size instances (C20 to C24) in less than 20 minutes. A total of 57 instances have been solved at the root node (before branching) and the average gap obtained at the root node is $1.21 \%$. The corresponding average gap with the B\&C algorithm in [6] was $3.37 \%$, and note that 5 instances could not be exactly solved.

Regarding the results obtained for the instances with more than 2 vehicles, reported in tables 7, 7 and 7, we have obtained 10 new optimal solutions for the ones with 3 vehicles, 19 with 4 vehicles and 13 with 5 vehicles. Also significant reductions in the average gaps can be observed. It should be noted that the difficulty of the instances increases with the number of vehicles in the case of the largest instances. However, this is not true for the smallest ones (sets C01, C11, C12 and C13), that get easier when the number of vehicles reaches a certain 
value. In fact, the number of optimally solved instances at the root node is 39,59 and 81 for 3,4 and 5 vehicles, respectively.

\begin{tabular}{rccc|cccrr}
\multicolumn{5}{c|}{ B\&C in [6] } & \multicolumn{5}{c}{ Enhanced B\&C } \\
\hline & $\begin{array}{r}\text { \# of } \\
\text { opt. }\end{array}$ & $\begin{array}{c}\text { Root node } \\
\text { gap }\end{array}$ & $\begin{array}{c}\text { Final } \\
\text { gap }\end{array}$ & $\begin{array}{c}\text { \# of } \\
\text { opt. }\end{array}$ & $\begin{array}{c}\text { Root node } \\
\text { gap }\end{array}$ & $\begin{array}{c}\text { Final } \\
\text { gap }\end{array}$ & $\begin{array}{r}\text { Time } \\
\text { (scs. })\end{array}$ & $\begin{array}{r}\text { B\&C } \\
\text { nodes }\end{array}$ \\
\hline C01 & 6 & 0.88 & - & 6 & 0.00 & - & 1.84 & 0.00 \\
C02 & 6 & 5.59 & - & 6 & 3.89 & - & 3.73 & 5.17 \\
C03 & 6 & 2.53 & - & 6 & 0.85 & - & 7.66 & 4.17 \\
C04 & 6 & 5.15 & - & 6 & 0.96 & - & 7.96 & 1.00 \\
C05 & 6 & 5.04 & - & 6 & 2.01 & - & 5.51 & 2.67 \\
C06 & 6 & 2.24 & - & 6 & 0.84 & - & 5.28 & 2.17 \\
C07 & 6 & 3.03 & - & 6 & 1.58 & - & 5.86 & 6.83 \\
C08 & 6 & 4.02 & - & 6 & 2.13 & - & 7.95 & 10.67 \\
C09 & 6 & 4.93 & - & 6 & 0.79 & - & 2.56 & 1.67 \\
C10 & 6 & 6.78 & - & 6 & 1.75 & - & 2.43 & 1.00 \\
C11 & 6 & 5.78 & - & 6 & 2.99 & - & 1.11 & 0.50 \\
C12 & 6 & 4.59 & - & 6 & 0.00 & - & 0.59 & 0.00 \\
C13 & 6 & 0.00 & - & 6 & 0.00 & - & 0.50 & 0.00 \\
C14 & 6 & 3.07 & - & 6 & 0.74 & - & 14.58 & 19.00 \\
C15 & 6 & 3.05 & - & 6 & 2.18 & - & 6.70 & 2.00 \\
C16 & 6 & 3.54 & - & 6 & 1.85 & - & 20.27 & 16.33 \\
C17 & 6 & 3.24 & - & 6 & 0.88 & - & 4.24 & 4.00 \\
C18 & 6 & 4.40 & - & 6 & 1.83 & - & 8.50 & 6.33 \\
C19 & 6 & 2.28 & - & 6 & 1.36 & - & 9.30 & 8.17 \\
C20 & 6 & 2.83 & - & 6 & 0.66 & - & 77.56 & 69.33 \\
C21 & 6 & 1.68 & - & 6 & 0.44 & - & 96.30 & 103.83 \\
C22 & 4 & 1.31 & 0.72 & 6 & 0.22 & - & 370.52 & 346.67 \\
C23 & 4 & 2.82 & 5.64 & 6 & 0.30 & - & 285.42 & 267.00 \\
C24 & 5 & 2.15 & 0.84 & 6 & 0.76 & - & 130.52 & 137.00 \\
\hline Global & 139 & 3.37 & 2.71 & 144 & 1.21 & 0.00 & & \\
\hline & & & & & & & &
\end{tabular}

Table 2: Computational results on the MM 2-WRPP instances

\section{Conclusions and future research directions}

In this paper we have continued the work on the MM $K$-WRPP started in [6]. We have improved the description of its associated polyhedron presenting new families of facet-inducing inequalities. One of the main contributions of this paper is that, in addition to inequalities involving variables associated with a single vehicle or all of them, we have presented new families of inequalities using variables corresponding to any subset of vehicles (called P-aggregate). Up to our knowledge, this is the first time that such type of inequalities has been introduced for multivehicle problems. Moreover, an enhanced branch-and-cut algorithm, based on the one presented in [6], has been implemented incorporating separation procedures for the new families of inequalities. The algorithm has been tested on the same set of instances used in 
the previous work and the computational results are very good and show the contribution of the new inequalities.

Regarding future work, we plan on finding under which conditions Honeycomb inequalities, presented in Section 3.2, are facet-inducing for $\operatorname{KWRPP}(G)$. We also want to study if there are aggregate, disaggregate and $\mathrm{P}$-aggregate versions of Path-Bridge inequalities from the WRPP that are valid and facet-inducing for the MM $K$-WRPP polyhedron. Finally, it would be interesting, from the computational point of view, to analyze the best way to choose the subsets of vehicles in order to find violated P-aggregate inequalities.

\begin{tabular}{rccc|cccrr}
\multicolumn{3}{c|}{ B\&C in [6] } & \multicolumn{6}{c}{ Enhanced B\&C } \\
\hline & $\begin{array}{r}\text { \# of } \\
\text { opt. }\end{array}$ & $\begin{array}{c}\text { Root node } \\
\text { gap }\end{array}$ & $\begin{array}{c}\text { Final } \\
\text { gap }\end{array}$ & $\begin{array}{l}\text { \# of } \\
\text { opt. }\end{array}$ & $\begin{array}{r}\text { Root node } \\
\text { gap }\end{array}$ & $\begin{array}{c}\text { Final } \\
\text { gap }\end{array}$ & $\begin{array}{r}\text { Time } \\
\text { (scs.) }\end{array}$ & $\begin{array}{r}\text { B\&C } \\
\text { nodes }\end{array}$ \\
\hline C01 & 6 & 0.00 & - & 6 & 0.00 & - & 1.60 & 0.00 \\
C02 & 6 & 10.56 & - & 6 & 7.04 & - & 5.12 & 8.33 \\
C03 & 6 & 7.06 & - & 6 & 3.86 & - & 17.68 & 9.67 \\
C04 & 6 & 7.27 & - & 6 & 4.08 & - & 12.78 & $2 ., 17$ \\
C05 & 6 & 7.63 & - & 6 & 7.09 & - & 6.15 & 3.50 \\
C06 & 6 & 7.29 & - & 6 & 4.16 & - & 14.17 & 9.83 \\
C07 & 6 & 5.81 & - & 6 & 4.07 & - & 21.07 & 37.33 \\
C08 & 6 & 7.47 & - & 6 & 5.07 & - & 17.72 & 26.00 \\
C09 & 6 & 7.85 & - & 6 & 2.08 & - & 5.04 & 9.83 \\
C10 & 6 & 5.20 & - & 6 & 1.51 & - & 2.58 & 2.00 \\
C11 & 6 & 3.58 & - & 6 & 0.00 & - & 0.90 & 0.00 \\
C12 & 6 & 3.50 & - & 6 & 0.44 & - & 0.84 & 0.17 \\
C13 & 6 & 0.00 & - & 6 & 0.00 & - & 0.52 & 0.00 \\
C14 & 6 & 7.08 & - & 6 & 4.81 & - & 35.89 & 37.67 \\
C15 & 6 & 4.61 & - & 6 & 2.96 & - & 12.95 & 9.67 \\
C16 & 5 & 7.63 & 4,92 & 6 & 4.56 & - & 103.20 & 153.67 \\
C17 & 6 & 6.03 & - & 6 & 4.44 & - & 9.43 & 7.33 \\
C18 & 6 & 4.66 & - & 6 & 2.68 & - & 9.51 & 4.17 \\
C19 & 6 & 4.67 & - & 6 & 2.31 & - & 32.10 & 35.33 \\
C20 & 3 & 6.20 & 5.22 & 4 & 2.85 & 0.84 & 192.70 & 150.25 \\
C21 & 1 & 11.27 & 11.59 & 3 & 2.58 & 0.40 & 345.62 & 255.33 \\
C22 & 0 & 5.78 & 5.56 & 3 & 1.14 & 0.61 & 524.27 & 271.00 \\
C23 & 0 & 5.47 & 5.32 & 2 & 1.54 & 0.60 & 1178.06 & 474.00 \\
C24 & 3 & 8.60 & 6.65 & 4 & 3.10 & 0.49 & 242.62 & 265.25 \\
\hline Global & 120 & 6.05 & 6.82 & 130 & 3.02 & 0.58 & & \\
\hline & & & & & & & &
\end{tabular}

Table 3: Computational results on the MM 3-WRPP instances

Acknowledgments: The authors wish to thank the Ministerio de Ciencia e Innovación of Spain (projects MTM2006-14961-C05-02 and MTM2009-14039-C06-02) for its support. 


\begin{tabular}{rccc|cccrr}
\multicolumn{3}{c|}{ B\&C in $[6]$} & \multicolumn{6}{c}{ Enhanced B\&C } \\
\hline & $\begin{array}{c}\text { \# of } \\
\text { opt. }\end{array}$ & $\begin{array}{c}\text { Root node } \\
\text { gap }\end{array}$ & $\begin{array}{c}\text { Final } \\
\text { gap }\end{array}$ & $\begin{array}{c}\text { \# of } \\
\text { opt. }\end{array}$ & $\begin{array}{c}\text { Root node } \\
\text { gap }\end{array}$ & $\begin{array}{c}\text { Final } \\
\text { gap }\end{array}$ & $\begin{array}{r}\text { Time } \\
\text { (scs. })\end{array}$ & $\begin{array}{r}\text { B\&C } \\
\text { nodes }\end{array}$ \\
\hline C01 & 6 & 0.00 & - & 6 & 0.00 & - & 1.47 & 0.00 \\
C02 & 6 & 11.95 & - & 6 & 6.69 & - & 6.26 & 7.00 \\
C03 & 6 & 5.64 & - & 6 & 5.16 & - & 27.45 & 27.50 \\
C04 & 6 & 6.66 & - & 6 & 3.34 & - & 29.09 & 69.83 \\
C05 & 6 & 2.52 & - & 6 & 2.44 & - & 7.80 & 7.67 \\
C06 & 6 & 6.09 & - & 6 & 3.29 & - & 30.81 & 55.17 \\
C07 & 6 & 6.20 & - & 6 & 4.93 & - & 39.40 & 64.83 \\
C08 & 4 & 12.05 & 12.52 & 6 & 7.08 & - & 53.65 & 87.00 \\
C09 & 6 & 5.18 & - & 6 & 2.27 & - & 4.52 & 1.00 \\
C10 & 6 & 3.95 & - & 6 & 1.66 & - & 2.23 & 0.33 \\
C11 & 6 & 3.84 & - & 6 & 0.00 & - & 0.86 & 0.00 \\
C12 & 6 & 4.23 & - & 6 & 0.00 & - & 0.55 & 0.00 \\
C13 & 6 & 0.00 & - & 6 & 0.00 & - & 0.53 & 0.00 \\
C14 & 5 & 9.11 & 10.22 & 6 & 5.69 & - & 542.08 & 626.50 \\
C15 & 6 & 2.83 & - & 6 & 1.93 & - & 20.65 & 28.00 \\
C16 & 0 & 11.40 & 9.45 & 6 & 6.95 & - & 299.04 & 380.00 \\
C17 & 6 & 4.27 & - & 6 & 3.12 & - & 8.87 & 5.83 \\
C18 & 6 & 1.25 & - & 6 & 1.20 & - & 8.61 & 0.83 \\
C19 & 6 & 4.07 & - & 6 & 3.22 & - & 42.82 & 44.83 \\
C20 & 0 & 15.37 & 15.14 & 4 & 5.79 & 1.57 & 844.76 & 293.50 \\
C21 & 0 & 18.57 & 18.40 & 3 & 4.64 & 1.82 & 1277.80 & 540.00 \\
C22 & 0 & 11.47 & 11.31 & 0 & 3.76 & 3.09 & - & - \\
C23 & 0 & 24.17 & 24.08 & 0 & 5.14 & 4.07 & - & - \\
C24 & 0 & 17.28 & 16.68 & 3 & 5.93 & 1.56 & 2046.87 & 1111.50 \\
\hline G10bal & 105 & 7.84 & 14.73 & 124 & 3.51 & 2.81 & & \\
\hline & & & & & & & &
\end{tabular}

Table 4: Computational results on the MM 4-WRPP instances 


\begin{tabular}{|c|c|c|c|c|c|c|c|c|}
\hline & \multicolumn{3}{|c|}{$\mathrm{B} \& \mathrm{C}$ in $[6]$} & \multicolumn{5}{|c|}{ Enhanced B\&C } \\
\hline & $\begin{array}{l}\text { \# of } \\
\text { opt. }\end{array}$ & $\begin{array}{l}\text { Root node } \\
\text { gap }\end{array}$ & $\begin{array}{c}\text { Final } \\
\text { gap }\end{array}$ & $\begin{array}{l}\text { \# of } \\
\text { opt. }\end{array}$ & $\begin{array}{l}\text { Root node } \\
\text { gap }\end{array}$ & $\begin{array}{c}\text { Final } \\
\text { gap }\end{array}$ & $\begin{array}{l}\text { Time } \\
\text { (scs.) }\end{array}$ & $\begin{array}{l}\text { B\&C } \\
\text { nodes }\end{array}$ \\
\hline $\mathrm{C} 01$ & 6 & 0.00 & - & 6 & 0.00 & - & 1.40 & 0.00 \\
\hline $\mathrm{C} 02$ & 6 & 10.61 & - & 6 & 3.60 & - & 4.61 & 4.33 \\
\hline $\mathrm{C} 03$ & 6 & 1.13 & - & 6 & 1.13 & - & 17.66 & 8.33 \\
\hline $\mathrm{C} 04$ & 6 & 6.99 & - & 6 & 3.29 & - & 18.15 & 34.33 \\
\hline $\mathrm{C} 05$ & 6 & 0.35 & - & 6 & 0.35 & - & 6.63 & 1.00 \\
\hline $\mathrm{C} 06$ & 5 & 3.90 & 6.62 & 6 & 0.86 & - & 76.70 & 98.00 \\
\hline $\mathrm{C} 07$ & 6 & 6.71 & - & 6 & 5.60 & - & 175.66 & 261.67 \\
\hline $\mathrm{C} 08$ & 4 & 13.98 & 20.81 & 6 & 4.43 & - & 149.16 & 301.00 \\
\hline C09 & 6 & 2.67 & - & 6 & 0.00 & - & 3.77 & 0.00 \\
\hline $\mathrm{C} 10$ & 6 & 3.61 & - & 6 & 1.59 & - & 2.04 & 0.33 \\
\hline C11 & 6 & 3.84 & - & 6 & 0.00 & - & 0.95 & 0.00 \\
\hline $\mathrm{C} 12$ & 6 & 4.23 & - & 6 & 0.00 & - & 0.59 & 0.00 \\
\hline $\mathrm{C} 13$ & 6 & 0.00 & - & 6 & 0.00 & - & 0.52 & 0.00 \\
\hline $\mathrm{C} 14$ & 3 & 8.48 & 10.51 & 5 & 4.52 & 1.49 & 270.47 & 225.60 \\
\hline $\mathrm{C} 15$ & 6 & 0.63 & - & 6 & 0.21 & - & 14.14 & 5.00 \\
\hline $\mathrm{C} 16$ & 1 & 13.35 & 15.13 & 2 & 6.36 & 4.54 & 317.03 & 205.50 \\
\hline $\mathrm{C} 17$ & 6 & 2.66 & - & 6 & 1.45 & - & 8.54 & 7.00 \\
\hline C18 & 6 & 0.00 & - & 6 & 0.00 & - & 8,31 & 0.00 \\
\hline C19 & 3 & 2.87 & 2.97 & 6 & 2.17 & - & 574.51 & 232.33 \\
\hline $\mathrm{C} 20$ & 0 & 30.01 & 20.92 & 1 & 8.70 & 5.20 & 245.88 & 72.00 \\
\hline $\mathrm{C} 21$ & 0 & 22.53 & 22.41 & 0 & 7.13 & 3.75 & - & - \\
\hline $\mathrm{C} 22$ & 0 & 23.48 & 23.39 & 0 & 5.69 & 5.08 & - & - \\
\hline $\mathrm{C} 23$ & 0 & 25.72 & 25.66 & 0 & 7.51 & 6.28 & - & - \\
\hline $\mathrm{C} 24$ & 0 & 25.06 & 24.80 & 3 & 9.63 & 5.23 & 1680.39 & 874.00 \\
\hline Global & 100 & 8.87 & 17.32 & 113 & 3.09 & 4.90 & & \\
\hline
\end{tabular}

Table 5: Computational results on the MM 5-WRPP instances 


\section{References}

[1] D. Ahr, Contributions to Multiple Postmen Problems. PhD Dissertation, University of Heidelberg, Germany, 2004.

[2] D. Ahr and G. Reinelt, New heuristics and lower bounds for the min-max $k$-Chinese Postman Problem. In: R. Mring, R. Raman, editors. Algorithms-ESA 2002, 10th Annual European Symposium, Rome, Italy, September 2002. Proceedings, Lecture Notes in Computer Science, vol. 2461. Berlin: Springer 2002, 64-74.

[3] D. Ahr and G. Reinelt, A Tabu search Algorithm for the Min-Max $k$-Chinese Postman Problem, Computers \& Operations Research 33 (2006), 3403-3422.

[4] D. Applegate, R.E. Bixby, V. Chvàtal and W. Cook, Finding cuts in the TSP, Technical report, DIMACS 95-05 (1995).

[5] A. Assad, W.L. Pearn and B. Golden, The Capacitated Chinese Postman Problem: Lower Bounds and Solvable Cases, American Journal of Mathematics and Management Science 7(1987), 63-88.

[6] E. Benavent, A. Corberán, I. Plana and J.M. Sanchis, Min-Max $K$-vehicles Windy Rural Postman Problem, Networks (2009), DOI: 10.1002/net 20334.

[7] E. Benavent, A. Corberán and J.M. Sanchis, An heuristic algorithm for the Min-Max $K$-vehicles Windy Rural Postman Problem, 2009. Submitted.

[8] A. Corberán, A. Letchford and J.M. Sanchis, A cutting plane algorithm for the General Routing Problem, Mathematical Programming 90 (2001), 291-316.

[9] A. Corberán, I. Plana and J. M. Sanchis, A Branch \& Cut Algorithm for the Windy General Routing Problem and special cases, Networks 49 (2007), 245-257.

[10] A. Corberán, I. Plana and J.M. Sanchis, The Windy General Routing Polyhedron: A global view of many known Arc Routing Polyhedra, SIAM J. Discrete Math. 22 (2008), 606-628.

[11] G. Desaulniers, Private communication, 2009.

[12] G. Frederickson, M. Hecht and C. Kim, Approximation algorithms for some routing problems, SIAM Journal on Computing 7 (1978), 178-193.

[13] G. Ghiani, D. Laganá, G. Laporte and R. Musmanno, A Branch-and-Cut Algorithm for the Undirected Capacitated Arc Routing Problem, Les Cahiers du GERARD, Université de Montréal, G-2007-39, 2007.

[14] W.L. Pearn, Solvable cases of the $k$-person Chinese postman problem, Operations Research Letters 16 (1994), 241-244,

[15] I. Plana, The Windy General Routing Problem. PhD Dissertation, University of Valencia, Spain, 2005. 